\title{
rtmpt: An $R$ package for fitting response-time extended multinomial processing tree models
}

\author{
Raphael Hartmann $^{1}$ (D) - Lea Johannsen ${ }^{1} \cdot$ Karl Christoph Klauer $^{1}$
}

Published online: 6 May 2020

(C) The Psychonomic Society, Inc. 2020

\begin{abstract}
Response-time extended multinomial processing tree models (RT-MPT; Klauer and Kellen, Journal of Mathematical Psychology, 82, 111-130 2018) provide estimates of process-completion times for cognitive processes modeled by means of multinomial processing tree (MPT) models (Batchelder and Riefer, Psychonomic Bulletin \& Review, 6, 57-86 1999). We present the $R$ package rtmpt with which it is possible to fit RT-MPT models easily. The package is free and open source, it can be used with two established MPT syntaxes, and has a number of useful features, such as suppressing processcompletion times for specific process outcomes, holding process probabilities constant, and changing some prior parameters. In the background of the $R$ package, an altered version of the original $C++$ code is used for the MCMC sampling. We provide a guide to using $r$ tmpt, validate the underlying hierarchical Bayesian algorithm of $r$ tmpt using simulation-based calibration and show that previously reported results can be reproduced using rtmpt.
\end{abstract}

Keywords $\mathrm{rtmpt} \cdot \mathrm{R}$ package $\cdot$ RT-MPT $\cdot$ Response time $\cdot$ Multinomial modeling $\cdot$ Hierarchical modeling .

Bayesian inference

For more than 150 years, researchers have tried to estimate completion times of cognitive processes. One of the earliest methods was proposed by Donders (1868) with his method of subtraction. Here, we consider a distant descendant of that method recently proposed by Klauer and Kellen (2018), called response-time extended multinomial processing trees (RT-MPTs). The method builds on the class of multinomial processing tree (MPT, Riefer \& Batchelder 1988) models (see Batchelder \& Riefer, 1999; Erdfelder et al., 2009; Hütter \& Klauer, 2016, for reviews).

MPTs model categorical frequencies and estimate the probabilities with which assumed latent processes occur. Adding response-time data, the RT-MPT extension permits the estimation not only of process probabilities but also of process-completion times of every process involved in an MPT. In addition, the time required for encoding task stimuli and motor execution of the response is estimated. To simplify reading, we refer to process-completion time as process time and to encoding plus motor execution time as

Raphael Hartmann

raphael.hartmann@psychologie.uni-freiburg.de

1 Department of Psychology, University of Freiburg, Engelbergerstrasse, 41, 79106 Freiburg, Germany motor time in what follows. The present article introduces and validates a software package called rtmpt for usage in the $R$ programming environment (R Core Team, 2019) that implements the RT-MPT method.

This package enables one to fit RT-MPTs to observed data and has several advantages over a previous implementation (Klauer \& Kellen, 2018). Most importantly, the previous implementation in $C++$ used the proprietary $C$ library NAG (The Numerical Algorithms Group (NAG), 2017), limiting its accessibility to researchers who have access to the required licenses. In contrast, rtmpt is completely free and open source and accessible to every interested researcher. NAG routines are replaced by routines from the $C$ library GSL (Galassi et al., 2018). Moreover, in rtmpt, we added the possibility to suppress the time of specific outcomes of a process with two possible outcomes. Suppressing a process time means setting the process time to zero and therefore holding it constant. Previously it was possible to suppress the time of either both outcomes of a process or none. Suppressing the time of only one outcome of a process can be useful whenever assuming a process either occurs with a certain probability or does not occur with the complementary probability such as the activation of association process in a process dissociation model (Jacoby, 1991). If activation does not occur, it often makes sense 
not to attach a time cost to this outcome. Furthermore, unlike the previous implementation, rtmpt can be easily used with established syntax for specifying MPT models such as the well-established EQN syntax (Hu, 1999; Heck et al., 2018a) or the one developed by Singmann and Kellen (2013) — referred to as $M D L$ syntax.

RT-MPTs have several advantages when compared to traditional MPT analyses. Adding response times has the benefit that the estimates of process probabilities can be expected to become more accurate. Moreover, incorporating response times into the analysis renders identified many MPT models that are not identified on the basis of the frequency data alone. For explanations of these two points, see Klauer and Kellen (2018).

Furthermore, as elaborated on below, traditional MPT models can be specified in equivalent variants, differing in the orders in which the involved processes are assumed to occur. These different variants of the same MPT model lead to different predictions for the response times. Thus, RT-MPTs allow one to distinguish between two or more such variants of the same MPT model. In other words, the order in which the processes are assumed to occur does matter for RTMPTs. This makes it possible to test two or more variantsor representations - of a traditional MPT model against each other (see Section "The RT-MPT model class").

The new model class RT-MPT can also be seen as an alternative to diffusion models (Ratcliff, 1978) in some instances. As illustrated by Klauer and Kellen (2018), RT-MPTs can produce comparable results. Additionally, traditional diffusion model analyses are restricted to twochoice paradigms, whereas RT-MPTs are not.

In this paper, we evaluate the newly developed $R$ package rtmpt by means of several approaches. A new procedure called simulation-based calibration (Talts et al., 2018) is used to validate the algorithm. This is one of the first times in psychology that simulation-based calibration has been used (see Section "Validation study (simulation-based calibration)"). A reproducibility study (Asendorpf et al., 2013) tests whether the results of analyses using the previous implementation can be reproduced. Finally, a small recovery study assesses the new features of the current

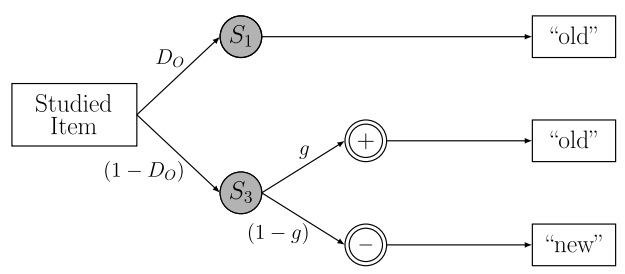

Fig. 1 2HTM (Detect-Guess variant). The rectangles indicate manifest states (i.e., item types and responses), and the gray circles indicate latent states $\left(S_{1}\right.$ and $S_{2}$ are states of certainty and $S_{3}$ is a state of uncertainty). The double circles indicate the outcomes of the guessing implementation, notably the suppression of individual process times.

In the next section, the RT-MPT model class is introduced more formally. We describe how MPTs are extended such that response latencies can be considered, what distributional assumptions are made, and discuss some of the advantages in more detail. Acknowledging that response-latency data are often too sparse to permit reliable estimation of the model parameters at the level of the individual participant, the rtmpt algorithm implements a hierarchical Bayesian model taking advantage of partial pooling of the individual data to obtain reliable group-level estimates while respecting and modeling any heterogeneity that may exist across participants. For a full description of the model class and estimation algorithm, interested readers are referred to the original paper by Klauer and Kellen (2018).

\section{The RT-MPT model class}

To understand this new RT-MPT model class, consider first the traditional MPT model class. In a nutshell, MPTs model the probabilities with which latent processes take place within a task (Erdfelder et al., 2009). Usually such tasks comprise two or more response alternatives. For instance, in a simple word recognition task there are two possible responses, "old" or "new", to indicate that a test word was either a word from a previously studied word list or not, respectively.

In using MPT models, assumptions are made about the processes believed to be involved in generating a given response. For example, one MPT model for the simple word recognition task is the popular two-high threshold model (2HTM; Snodgrass \& Corwin, 1988). It assumes that a word from the studied word list can either be detected as previously studied - through a detection process-or guessed as previously studied or not-through a guessing process. The same holds for new words; they are either detected as new words or guessed. An error can only occur by wrong guessing.

Figure 1 shows a graphical representation of the model in terms of its processing trees. Detection is assumed to play

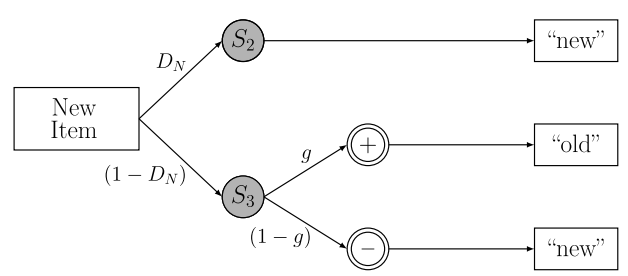

process where + denotes an "old" guess and - a "new" guess. The parameters $D_{O}, D_{N}$, and $g$ refer to the probabilities of detecting an old item as old, a new item as new, and guessing "old" 


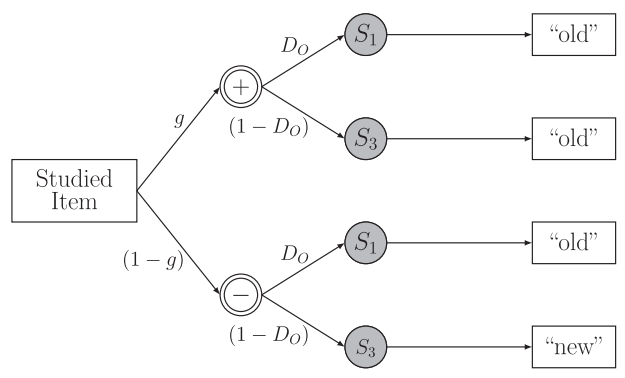

Fig. 2 2HTM (default-interventionist variant). The rectangles indicate manifest states (i.e., item types and responses), and the gray circles indicate latent states $\left(S_{1}\right.$ and $S_{2}$ are states of certainty and $S_{3}$ is a state of uncertainty). The double circles indicate the outcomes

a dominant role; if detection succeeds, the corresponding response is given ("old" for detecting a studied word with probability $D_{O}$ and "new" for detecting a new word with probability $D_{N}$ ). If detection fails (with probability $1-D_{O}$ or $1-D_{N}$ ), the response corresponding to the guessing outcome is given. With probability $g$, "old" is guessed and "new" with probability $1-g$.

Since MPT models are very flexible and relatively simple, they can be applied in many fields of psychology, such as recognition, source monitoring, immediate serial recall, implicit attitude measurement, and many more (for reviews see Erdfelder et al., 2009; Hütter \& Klauer, 2016). One shortcoming is, however, that the order in which the processes occur cannot be tested (Klauer \& Kellen, 2018). In most cases, several representations of one specific MPT model exist which are mathematically identical, but differ in the order and interaction of the processes in the graphical representation. For example, Fig. 1 shows only one variant of the 2HTM, which we call Detect-Guess. Another one, which leads to the same probability estimates, is shown in Fig. 2 and is called default-interventionist. In this variant of the model, guessing - or rather the activation of a bias in this case-takes place first and is overruled by the detection, if detection is successful. These two variants differ, however, in the predictions that they make for response times and hence give rise to different RT-MPT models.

Another shortcoming is that MPTs are often not identified (Klauer \& Kellen, 2018). Only by restraining some parameters to be equal can identifiability be achieved. For example, our running example, the Detect-guess variant of the 2HTM, is one of these unidentified MPTs. We have three parameters to estimate $\left(D_{O}, D_{N}, g\right)$, but only two equations; one for the hit rate and one for the false alarms (misses and correct rejections are redundant information). However, adding response times leads to an identified RTMPT model (Klauer \& Kellen, 2018).

There are two recent and prominent approaches that do consider RTs in the context of MPT models. The first

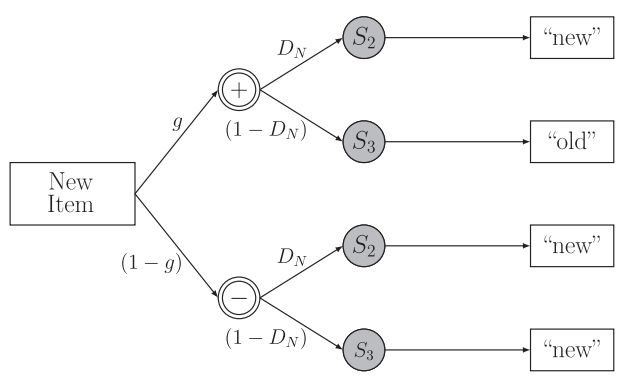

of the guessing process where + denotes an "old" guess and - a "new" guess. The parameters $D_{O}, D_{N}$, and $g$ refer to the probabilities of detecting an old item as old, a new item as new, and guessing "old"

approach was proposed by Heck and Erdfelder (2016) and the second by Klauer and Kellen (2018).

Heck \& Erdfelder's (2016) approach uses a nonparametric method to model the RTs alongside frequencies. In this context, Starns (2018) recently proposed a discreterace model that unlike (Heck \& Erdfelder, 2016) accommodates a speed-accuracy trade-off for the 2HTM (but see Heck \& Erdfelder, in press). Heck et al. (2018b) generalized Heck and Erdfelder's (2016) approach to handle not only RTs, but also many types of continuous data together with frequencies. This class of models is called generalized multinomial processing trees.

Klauer \& Kellen's (2018) approach is a hierarchical Bayesian approach and builds on the method of Klauer (2010). It makes explicit assumptions about the distributions of each process time and motor time, and combines RTs and categorical data into a joint likelihood function. As already mentioned, RT-MPTs provide estimates of process times and also overcomes a number of limitations of MPTs. Note, however, that these benefits come at the cost of additional distributional assumption for process and motor times.

To introduce RT-MPTs, we use the following notations. Let $p$ denote a process, $o$ its possible outcomes, $r$ the response, and $s$ the subject. In our 2HTM example, $p$ is either one of the detection processes or the guessing process, $o$ is success or failure for the detection processes and activation of a bias towards $r$, which is either "old" or "new". The probability parameters only depend on the process $p$ and the subject $s$. Therefore, we denote them with $\theta_{p, s}$. In our example $\boldsymbol{\theta}_{s}=\left(\theta_{D_{O}}, \theta_{D_{N}}, \theta_{g}\right)_{s}^{T}$.

Following $\mathrm{Hu}$ (2001), RT-MPTs assume that the observed response time is the sum of process times along the branch of the processing tree that generated the response and encoding plus motor execution time. Each process time is identified by its process name $p$ and its outcome $o$. Again,

\footnotetext{
${ }^{1}$ Note that we refer to person-level model parameters of MPT models by $\theta$ in the equations defining the model with a subscript identifying the process parameters referred to.
} 


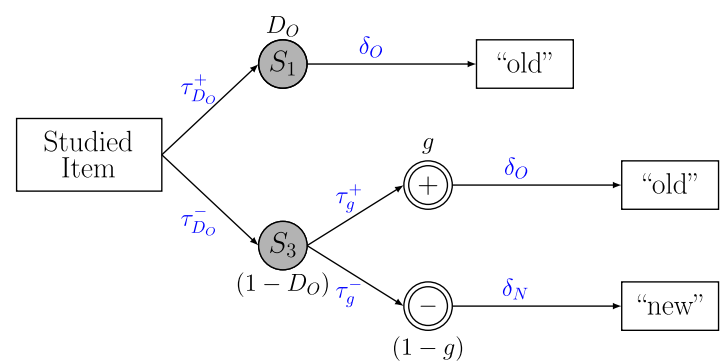

Fig. 3 Response-time extended 2HTM (Detect-guess variant). The rectangles indicate manifest states (i.e., item types and responses), and the gray circles indicate latent states $\left(S_{1}\right.$ and $S_{2}$ are states of certainty and $S_{3}$ is a state of uncertainty). The double circles indicate the outcomes of the guessing process where + denotes an "old" guess and - a "new" guess. The parameters $D_{O}, D_{N}$, and $g$ refer to

we have multiple subjects $s$ and therefore denote a process time by $\tau_{p, o, s}$. The motor times are denoted by $\delta_{r, s}$, indicating their possible dependency on the response and the subject. In our 2HTM or rather RT-2HTM (see Fig. 3) we have $\boldsymbol{\tau}_{s}=\left(\tau_{D_{N}}^{-}, \tau_{D_{N}}^{+}, \tau_{D_{O}}^{-}, \tau_{D_{O}}^{+}, \tau_{g}^{-}, \tau_{g}^{+}\right)_{s}^{T}$, where minus and plus signs denote the outcome (minus is either failure or a bias towards "new" and plus is either success or a bias towards "old") added as superscript, and $\boldsymbol{\delta}_{s}=\left(\delta_{O}, \delta_{N}\right)_{s}^{T}$, where $O$ and $N$ denote the two possible responses "old" and "new", respectively.

Further assumptions regard the distributions of the process and motor times. The process times are assumed to follow exponential distributions and the motor times normal distributions truncated from below at zero. Taken together, the person-level model comprises three groups of parameters: the process probabilities $\theta_{p, s}$, the parameters governing the distributions of the process times $\tau_{p, o, s}$, and the motor times $\delta_{r, s}$. Previously we stated the following assumptions:

$$
\begin{aligned}
\tau_{p, o, s} & \sim \operatorname{Exp}\left(\text { rate }=\lambda_{p, o, s}\right) \\
\delta_{r, s} & \sim \mathcal{T} \mathcal{N}\left(\text { lower }=0, \text { upper }=\infty, \mu=\gamma_{r, s}, \sigma=\sigma_{s}\right),
\end{aligned}
$$

where $\lambda_{p, o, s}$ are the corresponding rate parameters of the exponential distributions, and $\gamma_{r, s}$ and $\sigma_{s}$ the mean parameters and the standard deviation parameters, respectively, of the truncated normal distributions $\left(\mathcal{T N}\left(\gamma_{r, s}, \sigma_{s}, 0, \infty\right)\right)$. For the analysis, the process probabilities are probittransformed $\alpha_{p, s}=\Phi^{-1}\left(\theta_{p, s}\right)$, the rate parameters are used on the $\log$ scale $\beta_{p, o, s}=\log \left(\lambda_{p, o, s}\right)$, and the mean parameters $\gamma_{r, s}$ are used on the original scale. The (transformed) parameters are further decomposed into a grouplevel parameter $\mu$ and a subject-specific deviation from it (marked by a prime as superscript):

$$
\begin{aligned}
& \alpha_{p, s}=\mu_{p}^{(\alpha)}+\alpha_{p, s}^{\prime} \\
& \beta_{p, o, s}=\mu_{p, o}^{(\beta)}+\beta_{p, o, s}^{\prime} \\
& \gamma_{r, s}=\mu_{r}^{(\gamma)}+\gamma_{r, s}^{\prime}
\end{aligned}
$$

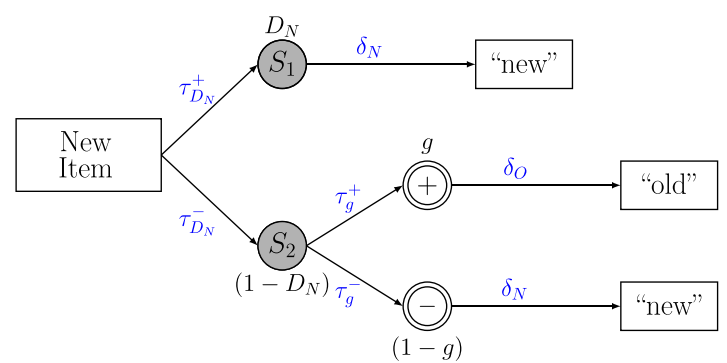

the probabilities of detecting an old item as old, a new item as new, and guessing "old". The $\tau$ parameters denote the time for a process (subscript) to complete for each outcome (superscript). The $\delta_{O}$ and $\delta_{N}$ parameters denote the time for encoding and motor execution for responding "old" and "new"

Klauer and Kellen (2018). The process-related parameters $(\alpha$ and $\beta$ ) are allowed to correlate with each other across persons and the motor-related parameters $(\gamma)$ are allowed to correlate with each other with variance-covariance matrices $\Sigma$ and $\Gamma$, respectively. Both variance-covariance matrices are estimated. For further details see Klauer and Kellen (2018).

\section{The $R$ package $r$ tmpt}

With the $R$ package rtmpt it is possible to fit RT-MPT models within the programming language $R$ (R Core Team, 2019). It uses an altered version of the $C++$ code by Klauer and Kellen (2018) in the background to sample posterior samples with a Metropolis-Gibbs sampler. This means, within $R$ the $C++$ program is called and executed, which returns quantities, such as the posterior samples to $R$. In the following, we describe how to use rtmpt, discuss an example (in Section "Example") as well as provide installation notes (in Section "Installation").

\section{Specifying models}

Specifying the MPT part of an RT-MPT model is possible by either using the well established EQN syntax (Hu, 1999; Heck et al., 2018a) for defining the MPT part of the RTMPT model or the $M D L$ syntax developed by Singmann and Kellen (2013). See Fig. 4 for the 2HTM written with the EQN syntax and Fig. 5 for the same model written with the $M D L$ syntax. Both syntaxes are mathematically equivalent. The difference is that for the $E Q N$ syntax each branch and its branch probability are specified on a separate line while for the $M D L$ syntax each response category and its probability are specified on a separate line. The probability of a response category is the sum of the branch probabilities of all branches leading to this category. As shown in Fig. 4, the EQN syntax separates tree, category, 


\begin{tabular}{|c|c|c|c|c|}
\hline \# Tree & & \# Category & ; & \# EQN \\
\hline Target & ; & hit & ; & Do \\
\hline Target & ; & hit & , & $(1-D o) * g$ \\
\hline Target & , & miss & ; & $(1-D o) *(1-g)$ \\
\hline Lure & ; & $f_{-} a$ & ; & $(1-D n) * g$ \\
\hline Lure & ; & $c_{-}^{-} r$ & ; & Dn \\
\hline Lure & & $c-r$ & ; & $(1-D n$ \\
\hline
\end{tabular}

Fig. 4 EQN syntax for the Detect-Guess variant of the Two-High Threshold model adapted from Heck et al. (2018a). $f_{-} a$ and $c_{-} r$ stand for false alarm and correct rejection, respectively. Do, Dn, and $g$ denote the process probabilities of detecting a target item, detecting a lure item, and guessing "old", respectively

and branch probability with semicolons. Note that in both types of syntax, it is important that the parameters of a branch occur in the order in which the branch is traversed from the root of the tree to the leaf as their order matters for RT-MPT models. The syntax of both types can be provided either directly as a character object in $R$ or by a text file. The former can be directly saved in an $R$ object while the latter must be provided by a path to the text file.

For both kinds of syntax, process probabilities can be set to constants by adding one further line in the file with the keyword constant and a colon following it. In our example, this line would look like this

constant: $g=.5$

if we want to set the guessing probability to .5. Likewise, the process times can be suppressed by providing the label of the process and its outcome, like

suppress: $9-, 9+$

if we want to suppress the process time of guessing in favor for "new" and the one for "old". Process time parameters cannot be set to arbitrary constants.

The EQN and $M D L$ syntax differ in how motor-time parameters are specified. For the $M D L$ style it is a little bit easier: after each line specifying the probabilities of a response category, write a semicolon and a response-label number indexing the associated motor time. The numbering

$$
\begin{aligned}
& \text { \# Target } \\
& \text { Do+(1-Do } * g \\
& (1-D o) *(1-g) \\
& \text { \# Lure } \\
& (1-D n) * g \\
& \text { Dn+(1-Dn)*(1-g) }
\end{aligned}
$$

Fig. $5 M D L$ syntax for the Detect-guess variant of the two-high threshold model. $D o, D n$, and $g$ denote the process probabilities of detecting a target item, detecting a lure item, and guessing "old", respectively

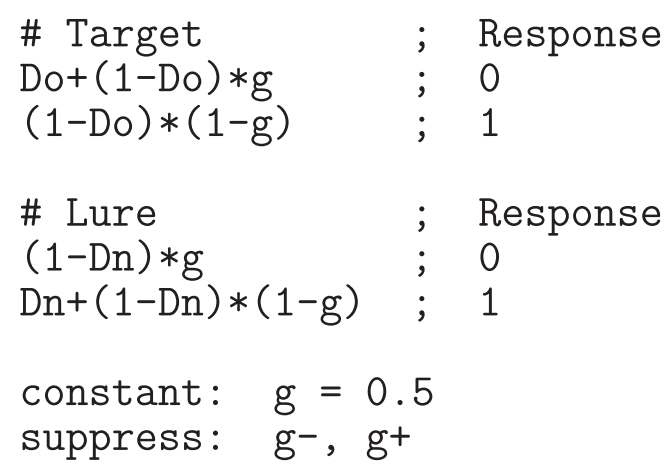

Fig. $6 M D L$ syntax for the Detect-guess variant of the two-high threshold model with constant guessing probability of .5 , suppressed process time of guessing for both outcomes and different motor times for the two possible responses. Do, Dn, and $g$ denote the process probabilities of detecting a target item, detecting a lure item, and guessing "old", respectively

should always include 0 . If there are two different motor time distributions, then each line has either a 0 or 1 after the semicolon. This implies that different motor times will be estimated for the responses associated with the categories marked 0 and the ones marked 1; see Fig. 6 for an example of the 2HTM with all restrictions and specifications written in the $M D L$ syntax. For the $E Q N$ syntax, one needs to specify the response mapping by means of a matrix with unique response categories using the keyword resp and a colon following it. For our 2HTM, the following lines need to be included if we assume different distributions of motor times for the "old" and "new" responses:

resp: Target ; hit ; 0
resp: Target ; miss ; 1
resp: Lure ; f_a $; 0$
resp: Lure ; c_r ; 1

The order of the lines does not matter, but the labels of the trees (i.e., "Target" vs. "Lure") and categories (i.e., "hit", "miss", "f_a", or "c_r") have to match the labels used in the data file. For an example of the 2HTM with all restrictions and specifications written in the EQN syntax see Fig. 7.

The to rtmpt model ( ) function needs to be applied in order to read and use the model. This function translates the syntax to a list of the class rtmpt model containing all the necessary information. For instance, the function call to rtmpt model (eqn file = eqn) is for using the EQN syntax with the character object (or path to the text file) named eqn. For the $M D L$ syntax use the argument mal file instead.

With the print () function it is possible to check the model specifications. It also provides some notes and additional information on how to interpret its output.

If the restrictions/specifications of the process probabilities, the process times, and/or the motor time labels are not 


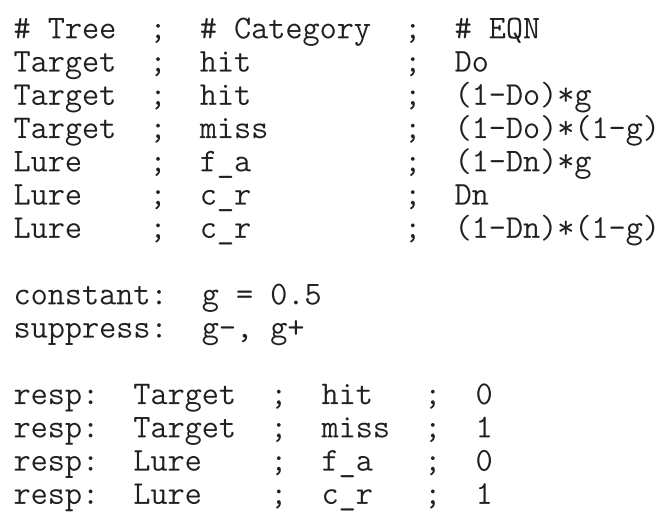

Fig. $7 E Q N$ syntax for the Detect-guess variant of the two-high threshold model adapted from Heck et al. (2018a) with constant guessing probability of .5 , suppressed process time of guessing for both outcomes and different motor times for the two possible responses. $D o, D n$, and $g$ denote the process probabilities of detecting a target item, detecting a lure item, and guessing "old", respectively

correct, the model object of the class rtmpt_model can be altered. For the process parameters the set_params () function can be used and for the motor time labels the set_resps ( ) function. Their use is further described in Appendix A.

\section{Data transformation}

The data must include five variables labeled subj, group, tree, cat, and $r t$ encoding, in order, the subject number, the experimental group the subject belongs to, the tree of the current trial, the observed response category, and the observed response time. The variable $r t$ must be provided in milliseconds. As previously mentioned the labels used in the model specification and the ones used in the data file must match. This is important if the data does not yet have the right format. For the main fitting function (see below) to work the data must only consist of numbers and except for $r t$ all variables must have values starting from zero. For instance, with a total of 50 subjects, all numbers from 0 up to 49 must be included in the variable subj. The function to rtmpt data () takes care of that. It takes the raw data and transforms it into a list of the class rtmpt data, which contains the transformed data as well as the transformations, which were used (old and new labels). In our previous example, we had two tree labels (Target and Lure) and four category labels (hit, miss, $\mathrm{f} a$, and $\mathrm{C} r$ ). These will be transformed into numbers according to the order in which they occur in the model file (Target $\rightarrow 0$, Lure $\rightarrow 1$, hit $\rightarrow 0$, miss $\rightarrow 1$, $\mathrm{f} a \rightarrow 2, \mathrm{C} r \rightarrow 3$ ). subj and group are transformed to numbers according to the order in which they occur in the raw data itself. For an example, see Section "Example".
The function to rtmpt data() takes two arguments, raw_data and model. raw_data must either be the path to a text file with the necessary label names in the header or a data.frame with the necessary variable names included in the column names. The parameter model must be of class rtmpt_model.

\section{Fitting models}

With the main function fit_rtmpt () of the $R$ package rtmpt it is possible to fit RT-MPT models to given data. A call of fit_rtmpt () does the MCMC sampling and returns the posterior samples. This function has two necessary arguments, model and data, and nine arguments with defaults.

The argument model must be a model list of class rtmpt_model. The argument data must either be the same as the raw_data in the to_rtmpt_data() function or the output of that function. Therefore it can be either a path to a file with a header, a data.frame or a data list rtmpt_data. If it is not a rtmpt_data object to_rtmpt_data ( ) will be used automatically. To keep track of the transformations done in this function it is, however, advisable to use it beforehand and use the output of it in the data argument.

Four of the arguments with defaults are the well known arguments for MCMC sampling, namely the number of chains $n$. chains, number of samples $n$. iter, number of warm-up samples $n$.burnin, and thinning n.thin. The thinning is conducted at the end of the MCMC sampling.

An additional argument Rhat_max specifies a threshold for the potential scale reduction factor $\hat{R}$ (Gelman \& Rubin, 1992). Once the maximal $\hat{R}$ of all the model parameters is (and stays) below this threshold, posterior samples are retained for the eventual output. ${ }^{2}$ Note that these values calculated on the run differ from the "a posteriori" $\hat{R}$, since the latter are calculated on the basis of only the retained posterior samples.

Another additional argument is Irep which gives the frequency of intermediate outputs. Every Irep samples the number Rhat max is recalculated and an intermediate output with $\hat{R}$ for the main parameters of the model as well as their current estimate is given.

In order to have some control over the priors, we included the prior params argument. A number of prior parameters can thereby be specified, controlling the informativeness of the prior distributions required for the hierarchical Bayesian approach. Note that it is not necessary to set these prior parameters, since all of them have default

\footnotetext{
${ }^{2}$ Because the actual posterior sampling starts after the threshold is reached, the number of iterations in the MCMC sampling step is always larger or equal to $\mathrm{n}$. thin $\times \mathrm{n}$. iter.
} 
values. These default values are comparable to those in Klauer and Kellen (2018), and they lead to rather broad prior distributions. The parameters controlling the prior distributions are explained in Appendix B. The last two arguments are indices and save_log_lik, which are both logical arguments, with functions explained in the next paragraph.

The output of the main function is a list consisting of at least the first three of the following objects.

1. samples: the MCMC samples saved as a mcmc.list class object of the $R$ package coda (Plummer et al., 2006).

2. diags: a list of diagnostics with the $95 \%$ and $99 \%$ highest density intervals and the medians of the main parameters. Furthermore, the "a posteriori" $\hat{R}$, the deviance information criterion (Spiegelhalter et al., 2002) are part of this list, as well as posterior predictive checks of the first type $\left(T_{1}\right.$; Klauer, 2010) for the frequencies as well as the latencies.

3. specs: a list of some specifications such as the model, the arguments of the function call, and the used data transformation.

4. indices: if indices = TRUE, the widely applicable information criterion (Watanabe, 2010) and leaveone-out cross-validation (Vehtari et al., 2017) saved in a list object.

5. LogLik: if save_log_lik = TRUE, the object LogLik containing the log-likelihood matrix of all samples and data points.

Table 1 gives an overview of all the functions. Note that only the first two are really necessary, but we recommend to use the remaining three for more control. For more detailed

Table 1 Overview of functions in rtmpt

\begin{tabular}{ll}
\hline Functions & Description \\
\hline fit_rtmpt: & Fit RT-MPT models. Main output: posterior \\
& samples. \\
to_rtmpt_model : & Convert model file (EQN or $M D L)$ to \\
& rtmpt_model. \\
to_rtmpt_data: & Convert data (text file or data.frame) to \\
& $\begin{array}{l}\text { rtmpt_model. } \\
\text { Restrict process parameters of an }\end{array}$ \\
set_params: & $\begin{array}{l}\text { rtmpt_model. } \\
\text { Specify number of different motor times in an }\end{array}$ \\
set_resps: & $\begin{array}{l}\text { rtmpt_model. } \\
\text { simulate data from an rtmpt_model object. }\end{array}$ \\
sim_rtmpt_data : & $\begin{array}{l}\text { Simulate data and fit model to that data } \\
\text { fit_rtmpt_SBC: }\end{array}$ \\
& $\begin{array}{l}\text { Siming the same priors. Main output: rank } \\
\text { statistic (of all parameters) for simulation- } \\
\text { based calibration. }\end{array}$
\end{tabular}

Table 2 First six lines of a fictive data frame usable with the rtmpt function fit_rtmpt ()

\begin{tabular}{llllll}
\hline & subj & cat & tree & group & rt \\
\hline 1 & 1 & miss & Target & 1 & 699 \\
2 & 1 & miss & Target & 1 & 655 \\
3 & 1 & c_r & Lure & 1 & 651 \\
4 & 1 & c_r & Lure & 1 & 676 \\
5 & 1 & hit & Target & 1 & 753 \\
6 & 1 & f_a & Lure & 1 & 1046 \\
\hline
\end{tabular}

descriptions check out the help pages of these functions, for example by typing ? fit_rtmpt, once rtmpt is installed.

\section{Example}

The first thing to do when trying to fit an RT-MPT to given data is to check what labels are used for cat and tree in the dataset. Table 2 shows how the first six lines of a data frame might look like. In order to find all labels of the two variable of interest one might execute these two lines

$$
\begin{aligned}
& \text { > unique (DF\$cat) } \\
& \text { > unique (DF\$tree) }
\end{aligned}
$$

which will give the two outputs

$$
\begin{aligned}
& \text { [1] "C_r" "f_a" "hit" "miss" } \\
& \text { [1] "Lure" "Target" }
\end{aligned}
$$

from which we can see the labels of the variables cat and tree, respectively.

If we wanted to use the $M D L$ syntax, we would have to change these labels to numbers by hand first. Since we can use labels in the $E Q N$ syntax, it might be more convenient to define our model file using that format. Figure 8 shows the EQN file for the 2HTM with one common detection parameter for old and new items. It is important that the labels match the ones used in the data frame.

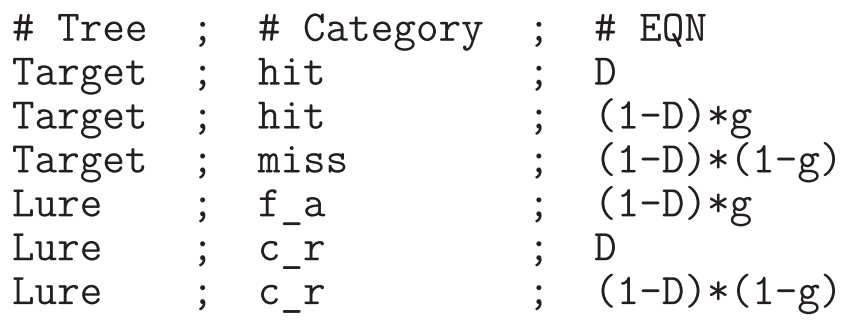

Fig. 8 Detect-guess variant of the restricted two-high threshold model (r2HTM.model) as EQN syntax. $D$ and $g$ denote the process probabilities of detecting an item correctly and guessing "old", respectively 
Once the model file is formulated we can transform it into an $R$ object of the class rtmpt_model by typing

$>$ model <- to_rtmpt_model (eqn_file="r2HTM.model ")

To see the details of this model, call the model object by model to get an output like in Listing 1 in Appendix C. This output contains four important details about the model. First, we see what the $M D L$ syntax of the model looks like, even if we defined the model with the EQN syntax. Remember, it was not possible to define it with the $M D L$ syntax, because we used labels instead of numbers in the data, but now that the mapping of the data and the model is done using the EQN syntax, the model can also be represented with the $M D L$ syntax. Second, it shows which of the process probability parameters will be estimated. Some notes and information about the parameters to estimate are also shown. Third, the process times to be estimated are shown. The latter two can be changed with the above mentioned function set_params ( ). Fourth, the response labels associated with specific motor times are shown. In the present state of the output in Listing 1 in Appendix C, all responses have the value 0 , thus for all responses the motor times are assumed to follow the same distribution.

Even though our model and our data have matching labels, we cannot use the dataset as it is currently. We need to transform it first. This is not a necessary step since the main function takes care of it, but it is prudent to perform it and check with

$>$ data <- to_rtmpt_data ( $\mathrm{DF}$, model)

$>$ data

The function call in the second line produces an output of the data transformation-or rather the mapping from the raw data to the one usable for the main function (see Listing 2 in Appendix C). This mapping was only possible because of the matching labels of data and model.

The RT-MPT model can now be fitted by calling the main function. Here we use 10,000 posterior samples, a warm-up of 1000 samples, a thinning of two, a very small threshold for the maximal $\hat{R}$ of 1.01 , an intermediate output after every 2000 samples, a prior precision $\epsilon$ of one, and choose to have WAIC and LOO as well.

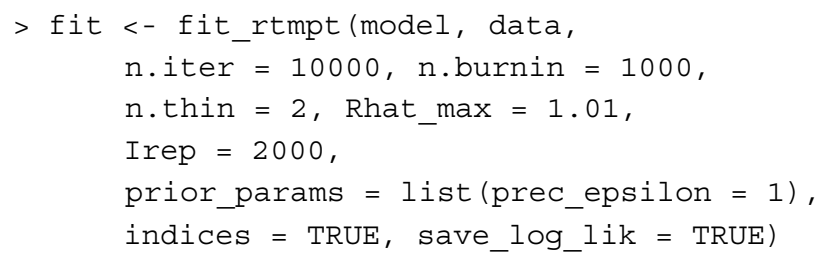

Note that a prior precision $\epsilon$ of one is included for demonstration purposes only. This is the default value and therefore will be used anyway if no other value is given. Executing the function starts the calculation of the initial values, then the sampling takes place. Intermediate outputs during the sampling show the progress by providing the $\hat{R}$ for the main parameters and the maximal $\hat{R}$ on the run. After the sampling a short output is given in the console, but all of it is saved in the $R$ object diags as well.

\section{Installation}

rtmpt is on the Comprehensive $\mathrm{R}$ Archive Network (CRAN) and can be installed by entering

$$
>\text { install.packages ("rtmpt") }
$$

in the command line of $R$. Alternatively, rtmpt can also be found on the software development platform GitHub. We provide instructions on how to install rtmpt on https://github.com/RaphaelHartmann/rtmpt.

\section{Reproducibility and simulation studies}

The $C++$ program underlying the package rtmpt has undergone many changes relative to the program used by Klauer and Kellen (2018). The most important change is that instead of the proprietary NAG library (The Numerical Algorithms Group (NAG), 2017), the open-source GSL library (Galassi et al., 2018) is used. In addition, some of the functions within the program are rewritten to make it more efficient. Additionally, the prior for the residual variance is changed from an improper prior to a proper gamma distribution. As just mentioned, it is now also possible to change certain prior parameters. Some minor changes include the routines for data and model-information input. All of these changes are potential sources for differences in the algorithm implemented by the program. Therefore, evaluating the program is desirable.

This is the purpose of the present section. The main part of this section is a simulation-based calibration (Talts et al., 2018) validating our implementation of the Bayesian algorithm. Next, we attempt to reproduce the major results of data analyses reported by Klauer and Kellen (2018) in their Tables 2 and 3 in a reproducibility study (Asendorpf et al., 2013). Finally, we present a small recovery study to test the partially new features of setting probability parameters constant and of suppressing individual process times.

\section{Validation study (simulation-based calibration)}

Simulation-based calibration (SBC) is a relatively new method for validating an algorithm for sampling from the 
posterior distribution of model parameters given the data (Talts et al., 2018). Its idea is that if we repeatedly sample parameters from the prior distribution of the assumed model, generate data from these parameters, and then fit the model to these generated datasets, the resulting posterior samples should follow the same distribution as the priors (Talts et al., 2018). Histograms of a rank statistic (see later) are used to check whether this holds true.

As argued by Talts et al. (2018), traditional recovery studies using a single set of ground truth values (i.e., a fixed set of prior values) to generate data are limited because they may lead us to conclude that recovery has been successful even for misspecified algorithms. Conversely, a posterior that appears biased away from the ground truth need not necessarily reflect a malfunctioning algorithm. SBC overcomes these limitations by considering the performance of the algorithm over the entire joint distribution of data and parameters.

SBC is based on the self-consistency condition

$p(\theta)=\iint p(\tilde{\theta}) p(\tilde{y} \mid \tilde{\theta}) p(\theta \mid \tilde{y}) \mathrm{d} \tilde{\theta} \mathrm{d} \tilde{y}$,

where $\tilde{\theta} \sim p(\theta)$ are the parameters-denoted as ground truth-sampled from the prior distribution and $\tilde{y} \sim p(y \mid \tilde{\theta})$ the data generated from the model using the ground truth. $\theta \sim p(\theta \mid \tilde{y})$ are the posterior samples.

The rank statistic mentioned above is defined as

$r\left(\left\{f\left(\theta_{1}\right), \ldots, f\left(\theta_{L}\right)\right\}, f(\tilde{\theta})\right)=\sum_{l=1}^{L} \mathbb{1}_{\left\{f\left(\theta_{l}\right)<f(\tilde{\theta})\right\}}$,

where $f: \Theta \rightarrow \mathbb{R}$ is a one-dimensional function (Talts et al., 2018). In our case $f$ is the function that picks one specific element of the posterior parameter vector $\theta_{l}$ or prior parameter vector $\tilde{\theta}$, meaning we calculate the rank statistic for each parameter separately.

By comparing the ground truth $\tilde{\theta}_{n}$ with the posterior samples $\left\{\theta_{n, 1}, \ldots \theta_{n, L}\right\}$ using the rank statistic (4) for each

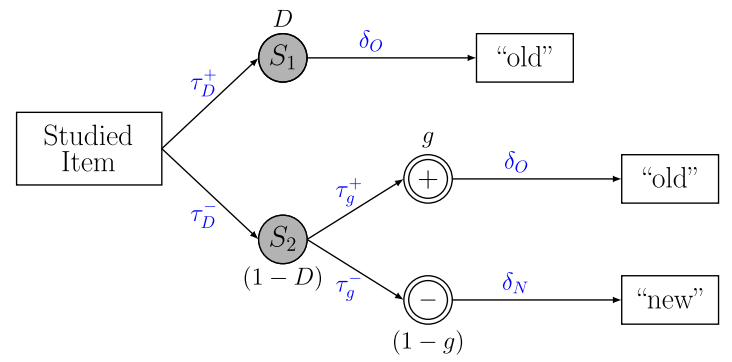

Fig. 9 Restricted response-time extended 2HTM (Detect-guess variant). The rectangles indicate manifest states (i.e., item types and responses), and the gray circles indicate latent states $\left(S_{1}\right.$ and $S_{2}$ are states of certainty and $S_{3}$ is a state of uncertainty). The double circles indicate the outcomes of the guessing process where + denotes an "old" guess and - a "new" guess. The parameters $D$, and $g$ refer replication $n \in\{1, \ldots, N\}$ we can test whether Eq. 3 holds true for one specific model $p(\tilde{y} \mid \tilde{\theta})$ (Talts et al., 2018). $L$ is the number of effective samples, meaning we thin the original posterior samples to reduce autocorrelation following Algorithm 2 of Talts et al. (2018). If the algorithm implemented through rtmpt is valid, this rank statistic is uniformly distributed within $[0, L]$. We test the uniformity via histograms and $\chi^{2}$-tests. As Talts et al. (2018) showed, the SBC procedure thereby allows the detection of many biases in the posterior samples such as autocorrelation, over- and underdispersion, and under- and overestimation. In fact, in our first attempt, we observed an overestimation of the mean process rates $e^{\mu^{(\beta)}}$. This was due to the fact that we forgot to apply the above-mentioned precision parameter prec_epsilon in the code for sampling from the conditional distribution of these parameters given the data and the other parameters - a rather subtle mistake that might well have gone unnoticed without this validation study.

We perform this test for the Detect-guess variant of a restricted version of the 2HTM (see Fig. 9) that is the running example for this manuscript (see Section "The RT-MPT model class"). As is often done in applications of the 2HTM, the restricted model assumes that detection for new and old items are equivalent processes so that we use the same parameters for their probability and process times. Using a simplified model helped to keep the computing time required for the simulations manageable. For the same reason, we adapted the priors to be more informative than the broad default priors. The default priors often lead to categories with zero cells and to extreme ranges of response times in the simulated data which tends to cause slow convergence of the algorithm. The adapted priors, detailed in Appendix D, lead to simulated datasets with more realistic category frequencies and response times.

With this model and the 2000 ground truth vectors $\tilde{\theta}$ sampled from the (altered) prior distributions, we generate 2000 datasets $\tilde{y}$, one for each $\tilde{\theta}$. Each dataset simulates 40

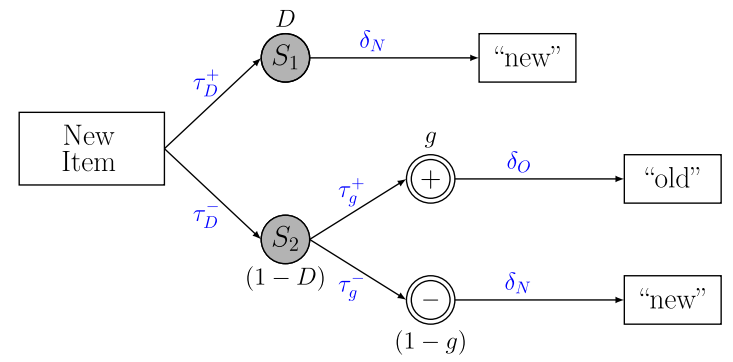

to the probabilities of detecting an item as what it is and guessing "old", respectively. The $\tau$ parameters denote the time for a process (in subscript) to complete for each outcome (in superscript). The $\delta_{O}$ and $\delta_{N}$ parameters denote the time for encoding and motor execution for responding "old" and "new" 
subjects responding to 30 trials per condition (studied items and new items). For each such dataset, we fit the model with four chains, each with 4000 samples, a warm-up of 200 samples, and an $\hat{R}$ threshold of 1.025 . For most of the 2000 datasets, a thinning of five was sufficient to have an effective sample size of at least 100 for the four times 4000 samples retained after thinning for each model parameter. If not, the fitting is repeated until the number of effective samples is at least 100 for each parameter. In cases where the number of effective sample size is still too low after three repetitions, we started the fitting anew with an increased thinning parameter of 15 .

The parameters we check consist of the group-level parameters $\left(\mu_{p}^{(\alpha)}, \mu_{p, o}^{(\beta)}, \mu_{r}^{(\gamma)}\right)^{T}$, the subject-specific deviations $\left(\alpha_{p, s}^{\prime}, \beta_{p, o, s}^{\prime}, \gamma_{r, s}^{\prime}\right)^{T}$, the variance-covariance matrices $\Sigma$ and $\Gamma$, the individual variances of the motor times $\sigma_{s}^{2}$, and the overall residual variance $\omega^{2}$. Since the subjects are replaceable, we only show the subject-specific SBC histograms for the first subject, meaning we exclude all subject-specific parameters other than $\left(\alpha_{p, 1}^{\prime}, \beta_{p, o, 1}^{\prime}, \gamma_{r, 1}^{\prime}\right)^{T}$ and $\sigma_{1}^{2}$, leading to a total of 42 parameters for which SBC histograms are shown. Histograms for all 393 parameters, including the other subject-specific parameters, are however reported in the OSF online materials.

Following the suggestions by Talts et al. (2018) we take $L=99$ effective samples from the posterior samples of the parameters and $N=2000$ replications (i.e., simulated datasets), such that we have a ratio of replications to bins of $20=2000 / 100$. Departing from Talts et al. (2018), we use two different approaches to take the 99 effective samples from the retained samples of the posterior distribution. First, the one also used by Talts et al. (2018), which is by thinning the retained samples to 99 , meaning, for the cases where our sample is of the size $4 \times 4000$ every $161^{\text {st }}$ sample is taken for the analysis. Second, we sample proportional to the effective sample size of each chain. This means that if one chain has clearly a higher minimal ${ }^{3}$ effective sample size than all others, more samples are taken from that chain than from the others.

The ranks of the samples of all 2000 replications cannot really be independent of each other, since knowing how many cases are in the first 99 ranks determines the number of cases in the $100^{\text {th }}$ rank. Therefore the check for uniformity of ranks via histogram is an oversimplification: The Pearson's Chi-squared test takes that into account. For each of the 393 parameters (including all subjectspecific parameters) we calculate the Chi-squared statistic with expected frequencies given by the expected uniform distribution (i.e., 20 per bin) and check how many of the

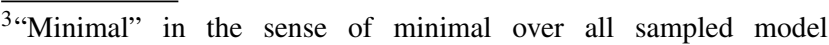
parameters.
}

parameters are above the critical value of $\chi^{2}(p=.95, d f=$ $99)=123.23$. The degrees of freedom are just the number of possible ranks minus one.

\section{Results}

Problems with fitting the 2000 simulated datasets occurred in a few cases with regard to two types of diagnostics, minimal effective sample size and convergence. First, the minimal effective sample size ranged from about ten to a few thousand, depending on the simulated dataset. For datasets with low minimal effective sample size (about $15 \%$ ), the thinning was increased from a factor of 5 to 15 . This not only increased the effective sample sizes, but also decreased $\hat{R}$. Second, 20 (1\%) of the datasets needed to be replaced because of severe convergence problems. About another 400 showed substantial differences between the "a posteriori" $\hat{R}$ and the threshold for $\hat{R}$ of the package for some of the sampled parameters, in which case fitting was repeated. Almost all of the cases with low minimal effective sample size are included in these 400 datasets.

Talts et al. (2018) propose to inspect histograms for systematic deviations from the uniform distribution. Figure 10 shows the histograms for the probit-transformed group-level process probability parameters $\mu^{(\alpha)}$ and for the group-level process rates $\mu^{(\beta)}$. Figure 11 shows the histograms of the rank statistic for the subject-specific deviations of the probit-transformed process probability parameters $\alpha^{\prime}$ and the subject-specific deviations of the process rates $\beta^{\prime}$. Figure 12 shows the histograms of the rank statistic for the variance and covariance parameters for the subject-specific deviations of the probit-transformed process probability parameters $\alpha^{\prime}$. The histograms of the rank statistic for the variance and covariance parameters for the subject-specific deviations of the process rates $\beta^{\prime}$ are shown in Fig. 13. Figure 14 shows the histograms of the rank statistic for the covariance parameters of the deviations of both the probit-transformed process probabilities and process rates.

Figure 15 shows the histograms of the rank statistic for the residual variance $\omega^{2}$, the variance of the motor times for the first subject $\sigma_{1}^{2}$, the group-level motor time mean parameter $\mu^{(\gamma)}$ for both responses, the subject-specific deviations of the motor times $\gamma^{\prime}$, and their variance and covariance parameters.

As can be seen, the histograms of the rank statistics show no deviations from the uniform distribution. This holds not only for the original sampling approach (proposed by Talts et al. 2018) for picking the 99 samples out of the generated 16,000 samples per dataset that underlies the shown histograms, but also for the proportional sampling method we used in addition. This means that there was no clear pattern in the histograms that indicate some kind of 
$\mu^{\alpha_{D}} \quad \chi^{2}=99.5$
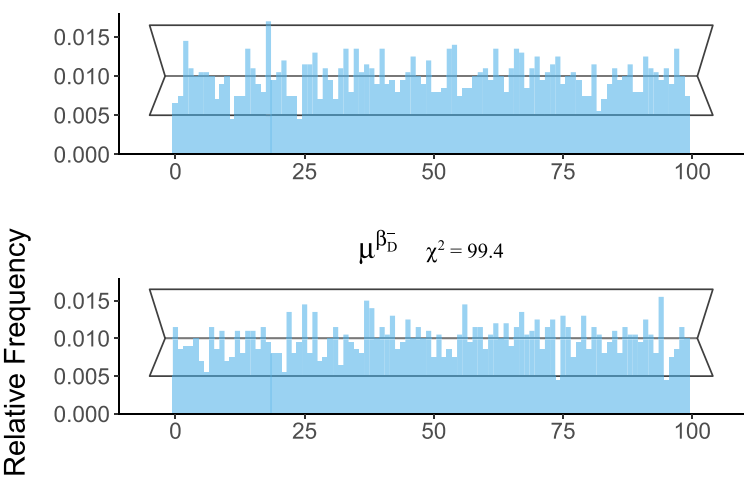

$\mu^{\beta_{D}^{+}} \quad \chi^{2}=98.5$

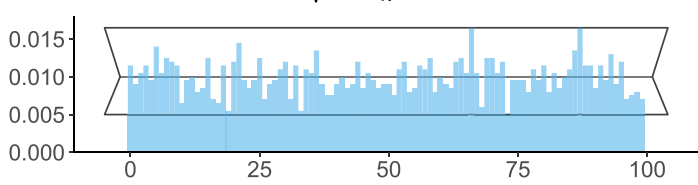

$\mu^{\alpha_{g}} \quad \chi^{2}=94.4$

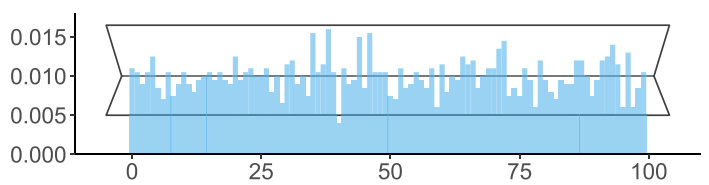

$\mu^{\beta_{\mathrm{g}}^{-}} \quad \chi^{2}=109.1$

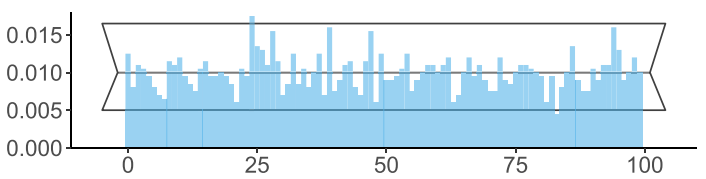

$\mu^{\beta_{g}^{+}} \quad \chi^{2}=89.1$

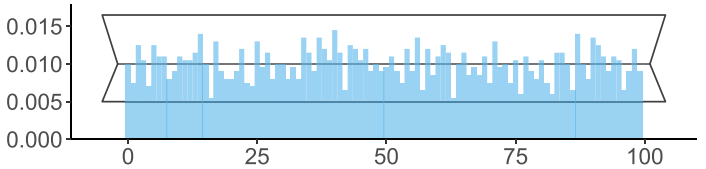

Rank Statistic

Fig. 10 Histograms of the rank statistic for the group-level processrelated parameters. On the horizontal axes are the rank statistics and on the vertical axes the relative frequencies (frequencies divided by $N=2000) . \mu^{\alpha}$ denote the group-level parameters referring to the probabilities on the probit scale and $\mu^{\beta}$ to the process rates. Lower and upper line specify the critical values of the exact two-tailed binomial test with $\alpha=.05$ for deviations between the observed and the expected frequency (middle line) of each individual bin. $D$ and $g$ denote the processes and - and + the outcomes for the process rates
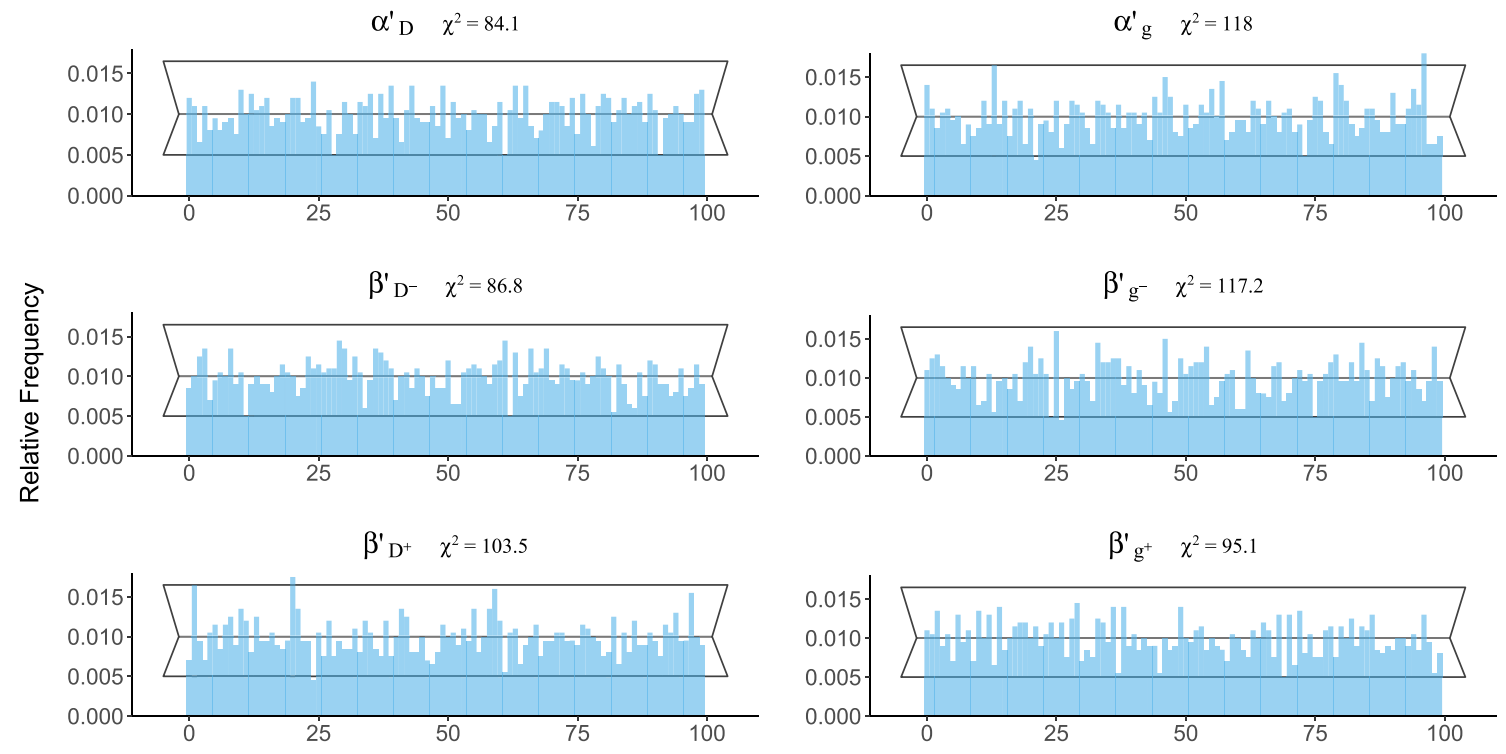

Rank Statistic

Fig. 11 Histograms of the rank statistic for the first subject's deviation for the process-related parameters. $\alpha^{\prime}$ denotes the deviation from the probit-transformed probabilities for the first subject and $\beta^{\prime}$ the deviation from the process rates for the first subject. $D$ and $g$ denote the processes and - and + the outcomes for the process rates; see also caption of Fig. 10 


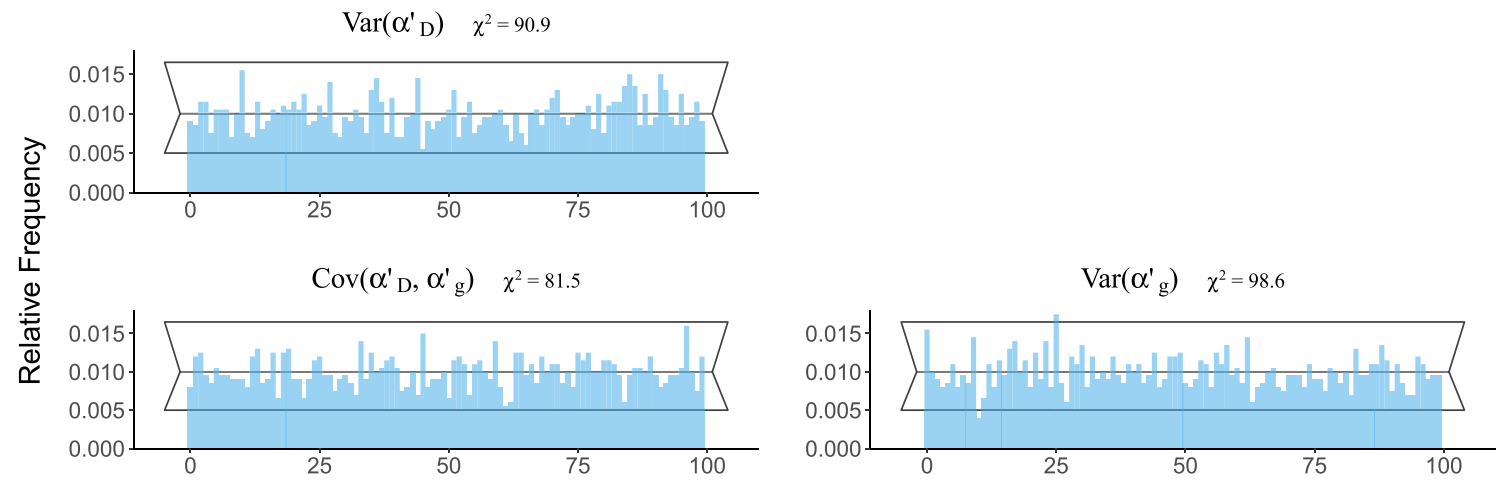

Rank Statistic

Fig. 12 Histograms of the rank statistic for the variance and covariance parameters of the subject-specific deviations from the probit-transformed probability parameters $\alpha^{\prime}$. D and $g$ denote the processes; see also caption of Fig. 10
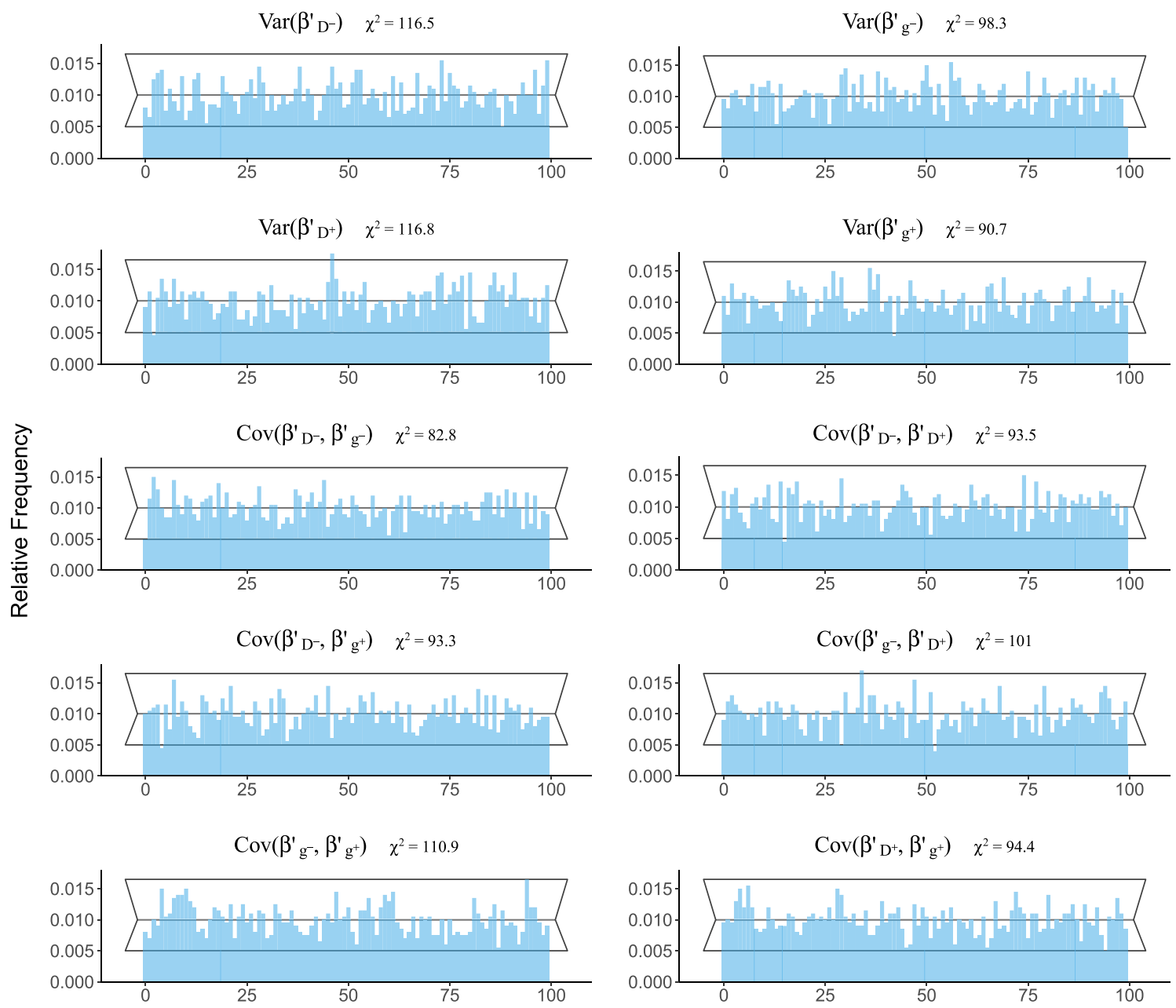

\section{Rank Statistic}

Fig. 13 Histograms of the rank statistic for the variance and covariance parameters of the subject-specific deviations from the process rate parameters $\beta^{\prime}$. D and $g$ denote the processes and - and + the outcomes for the process rates; see also caption of Fig. 10 

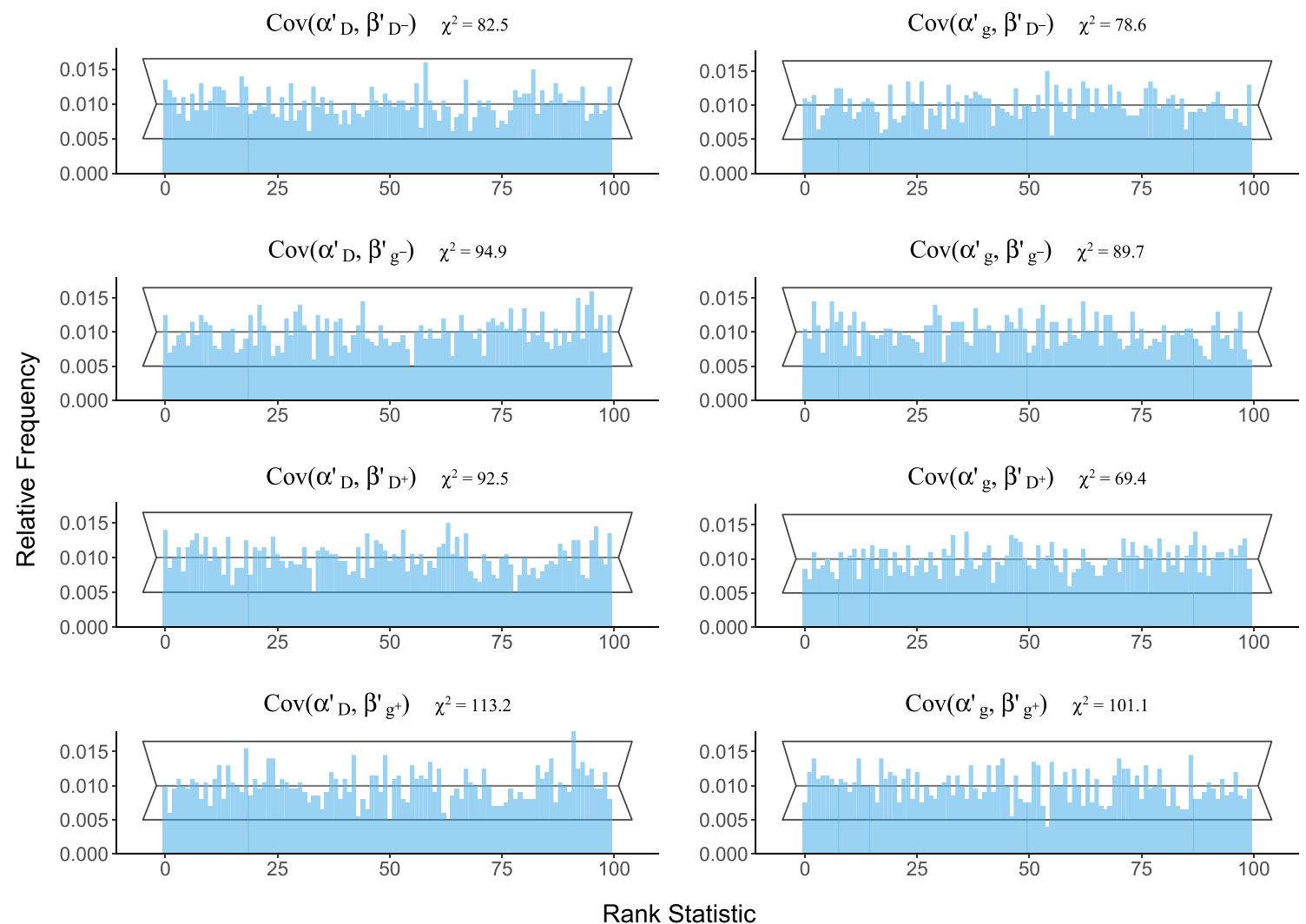

Fig. 14 Histograms of the rank statistic for the covariance parameters of the subject-specific deviations from both the probit-transformed process probability parameters and the process rate parameters. $D$ and $g$ denote the processes and - and + the outcomes for the process rates; see also caption of Fig. 10

bias-only some deviation from uniformity due to random fluctuations.

When using the original sampling method proposed by Talts et al. (2018), 4.07\% of the parameters show a Chisquare statistic above the critical value and $5.60 \%$ when the proportional sampling is used. Both values are close to the expected percentage of 5\% for Pearson's Chi-squared tests with an $\alpha$ level of .05. This means that the Chi-squared tests show little indication for substantial deviations from a uniform distribution of the ranks. ${ }^{4}$

Considering the histograms with Chi-squared values larger than the critical value, most of these occurred for the subject-specific parameters $\left(\alpha^{\prime}, \beta^{\prime}, \gamma^{\prime}, \sigma^{2}\right)$, which are the numerically largest class of parameters in the model. Under the proportional sampling procedure, there was also a variance and a covariance of the process-related parameters as well as one of the group-level motor times with high

\footnotetext{
${ }^{4}$ Note, however, that the $5 \%$ expectation strictly speaking holds for a set of independent Chi-squared tests, whereas the current Chi-squared tests are not independent from each other, because the different parameters are estimated from the same datasets.
}

Chi-squared values. Inspecting the corresponding histograms we can see no systematic pattern in the deviations, however. This suggests that these results just come from random fluctuations.

\section{Discussion}

No pattern in the histograms and about $5 \%$ of Chi-square statistics above the critical value suggest that the algorithm implemented by rtmpt passes Talts et al.'s (2018) strong and sensitive validity test. This is of course only true for the used model and needs to be validated anew for different models. In future versions of rtmpt we plan to implement the SBC approach, such that it can be used for every model.

The low minimal effective sample size of some of the posterior samples mentioned above reflects the liberal settings for fitting the model to the datasets chosen in the interest of keeping computing times for the simulation study at a manageable level. When analyzing individual experimental datasets, it is advised to specify more samples to be retained, higher thinning, and also a very low maximal $\hat{R}$, since the "a posteriori" $\hat{R}$ can differ substantially from the one calculated on the run. 

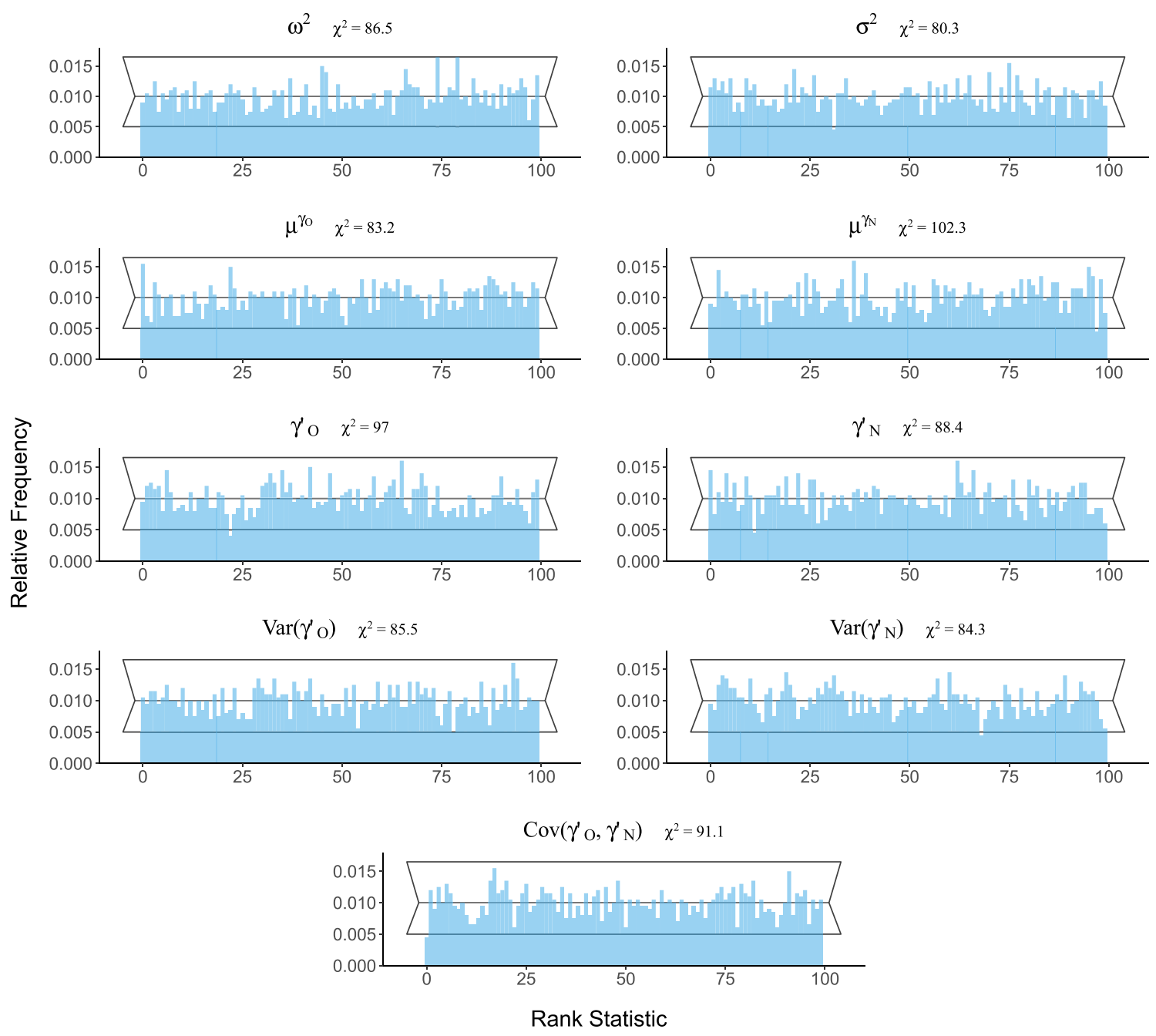

Fig. 15 Histograms of the rank statistic for the residual variance $\omega^{2}$, variance of the motor times for the first subject $\sigma^{2}$, group-level parameters for the motor times $\mu^{\gamma}$, deviation of the motor times for the

\section{Reproducibility study}

The goal of the reproducibility study is to test the reproducibility of the major results of the data analyses reported by Klauer and Kellen (2018) in their Tables 2 and 3. The datasets analyzed by Klauer and Kellen (2018) consist of the data from Arnold et al.'s (2015) Experiments 1, 2, and 3, as well as Dube et al.'s (2012) Experiments 1 and 2. In all of these studies, a word recognition task was used.

Arnold et al. (2015) used a base rate manipulation (change of ratio for old vs. new words) in the first experiment, a feedback manipulation (increase accuracy vs. increase speed) in the second experiment, and a manipulation of the frequency with which the words from the study list were presented during the study phase (half of them once and half of them twice) in the third experiment. Dube et al. (2012) conducted their first experiment with a mix of both, base rate and frequency manipulation. Their first subject $\gamma^{\prime}$, as well as variance and covariance parameters of the subject-specific deviations from the motor times. $O$ and $N$ refer to the response "old" and "new", respectively; see also caption of Fig. 10

second experiment was a mix of all three manipulations with a number of other minor modifications.

Parameter settings for the rtmpt fits mimic those employed by Klauer and Kellen (2018): We use four chains with 5000 samples each, an $\hat{R}$ threshold of 1.05 , and a thinning parameter of 11 . We use the same models as Klauer and Kellen (2018) for all the datasets, which are variations of the default-interventionist version of the 2HTM. Departing from Klauer and Kellen (2018), the prior for the residual variance is however changed from an improper prior to a gamma prior in rtmpt as already mentioned. For the gamma prior we use the default of the $R$ package, namely a mean of 0.005 and a variance of .01 .

\section{Results and discussion}

In Tables 3 and 4, we juxtapose the results from the rtmpt package and the original results reported by Klauer and 
Table 3 Comparison of parameter estimates between the newly developed $R$ package rtmpt and the old $C++$ program by Klauer and Kellen (2018)

\begin{tabular}{|c|c|c|c|c|c|c|c|c|c|}
\hline Par. & Prog. & $D_{N}$ & $D_{W}$ & $D_{S}$ & $g_{1}$ & $g_{2}$ & $g_{3}$ & $g_{4}$ & $g_{5}$ \\
\hline \multicolumn{10}{|l|}{ Exp. 1} \\
\hline \multirow[t]{2}{*}{$\mu^{(\alpha)}$} & rtmpt & $.50[.38, .63]$ & $.35[.27, .43]$ & $.79[.74, .85]$ & $.21[.14, .29]$ & $.28[.21, .36]$ & $.44[.35, .52]$ & $.57[.48, .65]$ & $.63[.57, .70]$ \\
\hline & $\mathrm{C}++$ & $.51[.38, .63]$ & $.35[.27, .43]$ & $.79[.74, .85]$ & $.21[.14, .29]$ & $.28[.21, .36]$ & $.44[.35, .52]$ & $.57[.48, .65]$ & $.63[.57, .70]$ \\
\hline \multirow[t]{2}{*}{$\mu_{-}^{(\beta)}$} & rtmpt & $203[159,248]$ & $190[154,211]$ & $160[116,211]$ & $19[10,31]$ & $63[33,101]$ & $113[66,158]$ & $116[67,174]$ & $133[85,185]$ \\
\hline & $\mathrm{C}++$ & $203[158,245]$ & $189[150,223]$ & $160[110,212]$ & $20[10,34]$ & $62[32,101]$ & $114[68,162]$ & $118[66,179]$ & $135[85,185]$ \\
\hline \multirow[t]{2}{*}{$\mu_{+}^{(\beta)}$} & rtmpt & $128[100,158]$ & $109[69,154]$ & $79[54,108]$ & $170[120,226]$ & $162[107,220]$ & $81[42,128]$ & $33[15,56]$ & $21[10,34]$ \\
\hline & $\mathrm{C}++$ & $127[98,156]$ & $107[67,157]$ & $78[51,107]$ & $169[115,226]$ & $163[101,223]$ & $79[40,127]$ & $33[15,57]$ & $20[10,35]$ \\
\hline \multicolumn{10}{|l|}{ Exp. 2} \\
\hline \multirow[t]{2}{*}{$\mu^{(\alpha)}$} & rtmpt & $.48[.34, .60]$ & $.21[.12, .30]$ & $.72[.63, .80]$ & $.19[.14, .24]$ & $.29[.23, .36]$ & $.47[.37, .56]$ & $.58[.50, .65]$ & $.65[.57, .71]$ \\
\hline & $\mathrm{C}++$ & $.48[.34, .61]$ & $.21[.12, .30]$ & $.72[.63, .80]$ & $.19[.14, .24]$ & $.29[.23, .36]$ & $.47[.38, .57]$ & $.58[.50, .65]$ & $.65[.58, .71]$ \\
\hline \multirow[t]{2}{*}{$\mu_{-}^{(\beta)}$} & rtmpt & $240[190,294]$ & $201[163,244]$ & $232[170,301]$ & $208[155,260]$ & $155[109,204]$ & $137[84,198]$ & $57[34,82]$ & $24[11,43]$ \\
\hline & $\mathrm{C}++$ & $238[188,293]$ & $199[162,242]$ & $227[165,298]$ & $205[157,255]$ & $155[112,203]$ & $136[86,193]$ & $58[35,84]$ & $24[12,42]$ \\
\hline \multirow[t]{2}{*}{$\mu_{+}^{(\beta)}$} & rtmpt & $152[110,195]$ & $104[62,153]$ & $100[71,130]$ & $177[128,232]$ & $208[165,260]$ & $88[50,130]$ & $50[27,75]$ & $17[9,32]$ \\
\hline & $\mathrm{C}++$ & $156[117,202]$ & $103[58,152]$ & $100[72,134]$ & $177[126,234]$ & $210[164,261]$ & $89[52,133]$ & $51[28,79]$ & $18[8,33]$ \\
\hline
\end{tabular}

Note. The table shows the median and 95\% HDIs (in square brackets) of the estimates for the Dube et al. (2012) datasets (two experiments) using the default-interventionist variant of the 2 HTM. Par. $=$ Parameter type. $\mu^{(\alpha)}$ are transformed to the probability scale and $\mu_{-}^{(\beta)}$ and $\mu_{+}^{(\beta)}$ to process times in milliseconds; Prog. = Program;

Kellen (2018) in their Tables 2 and 3. Tables 3 and 4 show the median of the posterior samples as well as the $95 \%$ highest density intervals in square brackets. As can be seen, the rtmpt and the original results are in close agreement. The largest differences occur for Experiment 2 by Arnold et al. (2015): One of its process probability estimates differs by about $47 \%$ and one of its process time estimates by about $17 \mathrm{~ms}$ between $r$ tmpt and the original results.

Because the posterior samples from the original results were still available, a more formal test of agreement between the two fits was possible. For this purpose, both sets of samples obtained for a given dataset were numbered from 1 to 20,000 . For each parameter, the difference in the values of given parameter was then computed for each pair of samples with equal numbers. We then checked whether the difference was credibly different from zero by computing 95\% highest density intervals for the 20,000 difference values and by checking whether the interval did not contain the value zero. None of the calculated differences in the posterior samples of all parameters are credibly different from zero: All 95\% highest density intervals include zero.

The numerically similar medians of the posterior samples and the highest density intervals for the differences of the posterior samples of both methods show that the new $\mathrm{C}++$ code in the background of rtmpt allows one to reproduce the fits produced by the original code.

\section{Recovery study}

Finally, we conducted a small recovery study to assess the adequacy of our implementation of the above-mentioned two features of the rtmpt package, namely holding process probabilities constant and suppressing specific process times. We report the goodness of recovery for models in which either of these features or both are implemented as well as for a model without these constraints. Our rationale for assessing these features is the following: If recovery performance is about as well for the constrained models as for the model without these constraints, this raises one's confidence that the implementation of the constraints is correct.

We use a model that is rather difficult to fit: The defaultinterventionist of the 2HTM (see Fig. 16) with two distinct detection processes $D_{O}$ and $D_{N}$ and a guessing process $g$. This model is difficult to fit because it is not identified in the setting of traditional MPTs, whereas it becomes identified only through the inclusion of response times (Klauer \& Kellen, 2018).

In total, we use six models from which to generate data. Model 6 is the model without restrictions. Models 1 to 5 implement combinations of the following three restrictions:

- holding $g$ constant at .5,

- suppressing the process time of guessing with outcome in favor for "new", 
Table 4 Comparison of parameter estimates between the newly developed $R$ package $r$ tmpt and the old $C++$ program by Klauer and Kellen (2018)

\begin{tabular}{|c|c|c|c|c|c|c|}
\hline \multirow[t]{2}{*}{ Par. } & \multirow[t]{2}{*}{ Prog. } & \multicolumn{2}{|l|}{ Exp. 1} & \multicolumn{2}{|l|}{ Exp. 2} & \multirow[t]{2}{*}{ Exp. 3} \\
\hline & & Low & High & Acc. & Speed & \\
\hline \multicolumn{7}{|c|}{ Process probabilities } \\
\hline \multirow[t]{2}{*}{$D_{N}$} & rtmpt & $.60[.31, .78]$ & $.55[.40, .68]$ & $.46[.11, .62]$ & $.47[.32, .61]$ & $.79[.72, .85]$ \\
\hline & $\mathrm{C}++$ & $.59[.28, .76]$ & $.53[.07, .67]$ & $.43[.06, .57]$ & $.45[.07, .59]$ & $.79[.73, .85]$ \\
\hline \multirow[t]{2}{*}{$D_{O}$} & rtmpt & $.33[.11, .53]$ & $.14[.01, .37]$ & $.26[.12, .50]$ & $.06[.00, .24]$ & $.02[.00, .11] ; .55[.42, .67]^{a}$ \\
\hline & $\mathrm{C}++$ & $.34[.14, .52]$ & $.15[.00, .58]$ & $.28[.13, .55]$ & $.10[.00, .40]$ & $.01[.00, .09] ; .54[.43, .68]^{a}$ \\
\hline \multirow[t]{2}{*}{$g$} & rtmpt & $.39[.20, .55]$ & $.66[.52, .75]$ & $.57[.35, .67]$ & $.58[.46, .67]$ & $.57[.48, .66]$ \\
\hline & $\mathrm{C}++$ & $.38[.20, .52]$ & $.65[.30, .74]$ & $.55[.30, .65]$ & $.56[.29, .64]$ & $.58[.49, .66]$ \\
\hline \multicolumn{7}{|c|}{ Process times in ms for negative outcomes } \\
\hline \multirow[t]{2}{*}{$D_{N}$} & rtmpt & $240[197,288]$ & $306[239,366]$ & $476[386,574]$ & $87[51,113]$ & $174[130,220]$ \\
\hline & $\mathrm{C}++$ & $237[187,287]$ & $302[83,363]$ & $465[362,568]$ & $83[39,109]$ & $178[136,224]$ \\
\hline \multirow[t]{2}{*}{$D_{O}$} & rtmpt & $165[110,240]$ & $255[219,294]$ & $451[380,522]$ & $88[58,110]$ & $137[114,160] ; 153[123,182]^{\mathrm{a}}$ \\
\hline & $\mathrm{C}++$ & $164[49,191]$ & $250[97,291]$ & $448[377,532]$ & $86[43,109]^{\mathrm{c}}$ & $138[116,159] ; 155[127,183]^{\mathrm{a}, \mathrm{b}}$ \\
\hline \multirow[t]{2}{*}{$g$} & rtmpt & $135[55,199]$ & $94[57,131]$ & $47[20,75]$ & $46[17,80]$ & $55[28,87]$ \\
\hline & $\mathrm{C}++$ & $136[49,191]$ & $97[57,245]$ & $49[16,81]$ & $45[22,90]$ & $55[28,81]$ \\
\hline \multicolumn{7}{|c|}{ Process times in ms for positive outcomes } \\
\hline \multirow[t]{2}{*}{$D_{N}$} & rtmpt & $71[41,122]$ & $206[150,259]$ & $312[151,388]$ & $78[53,101]$ & $81[59,104]$ \\
\hline & $\mathrm{C}++$ & $70[35,118]$ & $202[39,258]$ & $295[129,385]$ & $77[49,102]$ & $81[61,104]$ \\
\hline \multirow[t]{2}{*}{$D_{O}$} & rtmpt & $84[30,156]$ & $63[20,134]$ & $160[78,282]$ & $57[23,113]$ & $91[21,207] ; 59[35,85]^{\mathrm{a}}$ \\
\hline & $\mathrm{C}++$ & $87[34,160]$ & $59[16,166]$ & $168[84,305]$ & $59[20,98]$ & $84[17,203] ; 56[35,83]^{a, b}$ \\
\hline \multirow[t]{2}{*}{$g$} & rtmpt & $168[122,213]$ & $77[34,143]$ & $92[47,152]$ & $67[37,98]$ & $89[69,110]$ \\
\hline & $\mathrm{C}++$ & $171[119,216]$ & $76[37,287]$ & $100[55,197]$ & $69[40,106]$ & $88[68,107]$ \\
\hline \multicolumn{7}{|c|}{ Motor times } \\
\hline \multirow[t]{2}{*}{$N$} & rtmpt & $622[597,647]$ & $682[655,710]$ & $770[729,815]$ & $552[509,591]$ & $620[589,650]$ \\
\hline & $\mathrm{C}++$ & $623[597,650]$ & $684[652,711]$ & $773[722,819]$ & $551[510,591]$ & $620[593,648]$ \\
\hline \multirow[t]{2}{*}{$O$} & rtmpt & $636[612,662]$ & $592[566,617]$ & $716[671,763]$ & $518[477,562]$ & $582[559,605]$ \\
\hline & $\mathrm{C}++$ & $638[613,663]$ & $593[568,618]$ & $716[667,765]$ & $517[475,560]$ & $583[560,607]$ \\
\hline
\end{tabular}

Note. The table shows the median and 95\% HDIs (in square brackets) of the estimates for the Arnold et al. (2015) datasets (three experiments) using the default-interventionist variant of the 2HTM. Par. = Parameter: For the process parameters this is the process name and for the motor times the response; Prog. = Program; Low = Low base-rate; High = Highbase-rate; Acc. = Accuracy;

${ }^{a}$ Values for weak and strong studied items, respectively

${ }^{\mathrm{b}}$ The order of weak and strong were mistakenly switched in Table 2 of Klauer and Kellen (2018)

${ }^{\mathrm{c}}$ The values from Table 2 in Klauer and Kellen (2018) were mistakenly reported as 168[84,304]

- suppressing the process time for both outcomes of the guessing process.

This leads to three models with constant $g$, which are actually identified on the basis of the response frequencies alone (Models 1 to 3), and to two models with only one guessing-process outcome time suppressed (Models 2 and 4 ), and two models with both guessing-process outcome times suppressed (Models 3 and 5). The $M D L$ file for the model with all restrictions (Model 3) can be seen in Fig. 17.
For each model, we generate 100 datasets with 40 participants and 30 trials per tree (studied item and new item) and participant. Note that the small size of these datasets limits the precision with which parameters can be estimated so that the overall goodness of recovery cannot be expected to be excellent.

Ground-truth values for the group-level parameters were chosen from fits to real data in order to generate realistic datasets: Thus, for the variance-covariance matrices $\Sigma$ and $\Gamma$, and for the residual variance $\omega^{2}$, we directly use the 


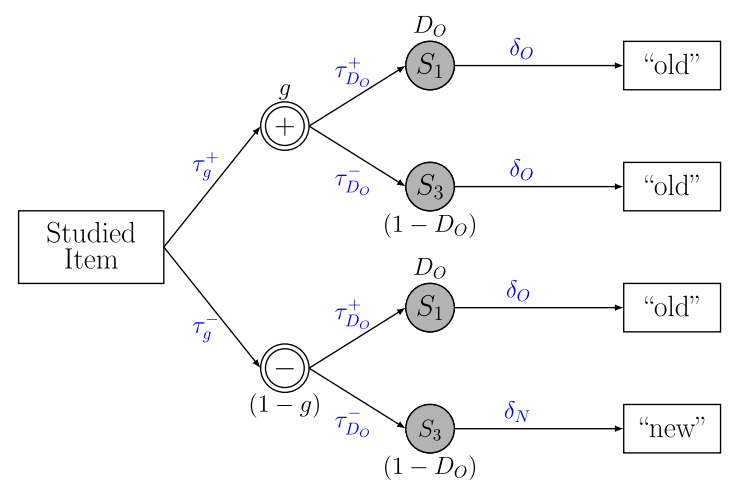

Fig. 16 Response-time extended 2HTM (default-interventionist variant). The rectangles indicate manifest states (i.e., item types and responses), and the gray circles indicate latent states $\left(S_{1}\right.$ and $S_{2}$ are states of certainty and $S_{3}$ is a state of uncertainty). The double circles indicate the outcomes of the guessing process where + denotes an "old" guess and - a "new" guess. The parameters $D_{O}, D_{N}$, and $g$

median of the rounded parameter estimates from fitting the default-interventionist variant of the 2HTM to the data of the first experiment by Arnold et al. (2015). The subjectspecific deviations are sampled from a normal distribution with mean zero and variance-covariance matrices $\Sigma$ or $\Gamma$, respectively. The subject-specific variances of the motor times $\sigma_{s}^{2}$ are sampled from a scaled inverse Chisquared distribution with 10 degrees of freedom and $\omega^{2}$ as scale parameter. This number of degrees of freedom was necessary, since using the default of 2 leads to many RTs with unrealistic values larger than $3500 \mathrm{~ms}$.

Each model was fit to the 100 datasets generated from that model. Except for the degrees of freedom of the scaled inverse Chi-squared distribution (10) of $\sigma^{2}$, the default priors of the rtmpt package are used. For each dataset, we fit the corresponding model with four chains, 4000 samples per chain, a thinning parameter of five, and an $\hat{R}$ threshold of 1.025 .

$$
\begin{aligned}
& \text { \# Target }
\end{aligned}
$$

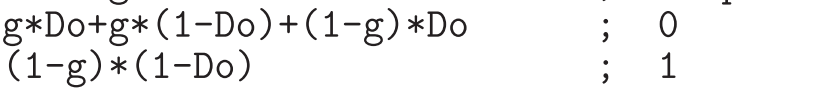

$$
\begin{aligned}
& \text { \# Lure } \\
& \mathrm{g} *(1-\mathrm{Dn}) \\
& \mathrm{g} * \mathrm{Dn}+(1-\mathrm{g}) * \mathrm{Dn}+(1-\mathrm{g}) *(1-\mathrm{Dn}) \quad ; \quad 1 \\
& \text { constant: } g=0.5 \\
& \text { suppress: } \mathrm{g}^{-}, \mathrm{g}^{+}
\end{aligned}
$$

Fig. $17 M D L$ syntax for the default-interventionist variant of the two-high threshold model with constant guessing probability of .5 , suppressed process time of guessing for both outcomes and different motor times for the two possible responses

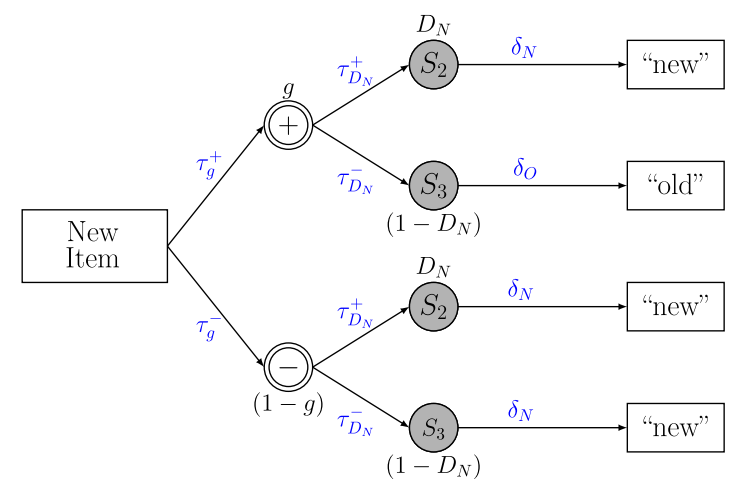

refer to the probabilities of detecting an old item as old, a new item as new, and guessing "old". The $\tau$ parameters denote the time for a process (subscript) to complete for each outcome (superscript). The $\delta_{O}$ and $\delta_{N}$ parameters denote the time for encoding and motor execution for responding "old" and "new"

In order to measure the recovery precision, we used the root mean squared error (RMSE) and the bias. The RMSE of an estimator $\hat{\theta}$ for a random variable $\theta$ is defined as

$\operatorname{RMSE}(\hat{\theta})=\sqrt{\frac{1}{R} \sum_{r=1}^{R}\left(\hat{\theta}_{r}-\theta_{r}\right)^{2},}$

and the bias as

$\operatorname{bias}(\hat{\theta})=\frac{1}{R} \sum_{r=1}^{R}\left(\hat{\theta}_{r}-\theta_{r}\right)$,

where $R$ denotes the number of replications, $\hat{\theta}_{r}$ the mean of the posterior samples, and $\theta_{r}$ the true value for the $r^{t h}$ replication.

\section{Results and discussion}

Table 5 shows the parameter recovery for all models. The estimates of the process probability parameters for the first three models are quite precise. The means of the posterior deviate from the true values by only about $1 \%$. The RMSEs support that; the largest value being 0.06 . The mean of the estimated process times is mostly also close to the true value. Typically, they differ from the true values by less than $10 \mathrm{~ms}$. The largest difference is about $32 \mathrm{~ms}$ and the largest RMSE value is .045. Most process-related parameter estimates (18 of 21) have a slightly negative bias, indicating that the true values are slightly underestimated. ${ }^{5}$

\footnotetext{
${ }^{5}$ Considering process time-related parameters, the (relatively) largest cases of bias occur for the largest true values (such as 0.24 for $\left.\mu_{\left(D_{N},-\right)}^{\beta}\right)$, suggesting that they to some extent reflect shrinkage towards the mean $(0.1)$ of their somewhat informative prior.
} 
Table 5 Parameter recovery study: Group-level means of process-related parameters

\begin{tabular}{lllllll}
\hline Par. & Proc. & True & Est. & RMSE & SE & SD
\end{tabular}

Model 1

$\mu^{\alpha}$

$D_{N}$

0.60

0.59

$-0.0066$

0.0045

$\mu_{-}^{\beta}$

$D_{O}$

0.35

0.34

0.045

$-0.0121$

0.0060

0.05

0.20

$-0.0330$

0.0032

$-0.0194$

0.0022

0.04

$D_{O}$

0.16

0.029

0.14

0.023

$\mu_{+}^{\beta}$

0.14

0.0052

0.0023

0.03

$D_{N}$

0.07

$-0.0065$

0.0016

0.02

$D_{O}$

0.08

$-0.0074$

0.0022

0.03

g

0.17

0.022

0.0002

0.0022

0.03

Model 2

$\begin{array}{lll}\mu^{\alpha} & D_{N} & 0.60 \\ \mu_{-}^{\beta} & D_{O} & 0.35 \\ \mu_{+}^{\beta} & D_{N} & 0.24 \\ & D_{O} & 0.16 \\ & D_{N} & 0.07 \\ & D_{O} & 0.08 \\ & g & 0.17\end{array}$

0.59

$-0.0095$

0.0050

0.05

$-0.0034$

0.0047

0.05

0.22

$-0.0149$

0.0024

0.03

$-0.0031$

0.0014

0.01

0.07

$-0.0004$

0.0013

0.01

0.024

0.0022

0.02

0.17

$-0.0097$

0.0019

0.02

Model 3

$\mu^{\alpha}$

$D_{N}$

0.60

0.60

$-0.0029$

0.051

$-0.0054$

0.0052

0.05

$\mu_{-}^{\beta}$

0.24

0.34

0.049

$D_{N}$

0.23

$-0.0048$

0.0050

0.05

$D_{O}$

0.012

$\mu_{+}^{\beta}$

0.16

$-0.0030$

0.0021

0.02

0.16

0.0012

0.01

$-0.0015$

0.0010

0.01

$D_{O}$

0.08

0.08

$-0.0067$

0.0014

0.02

Model 4

$\mu^{\alpha}$

$D_{N}$

0.60

0.54

$-0.0559$

0.0081

0.11

$D_{O}$

0.35

0.098
0.065

$-0.0023$

0.0067

0.08

0.38

0.060

$-0.0233$

0.0058

0.07

0.24

$-0.0127$

0.0028

0.03

0.16

0.012

$-0.0031$

0.0012

0.01

0.0031

0.0015

0.02

$0.09-0.024$

0.0100

0.0024

0.03

0.16

0.024

$-0.0109$

0.0021

0.03

Model 5

$\begin{array}{lllll}\mu^{\alpha} & D_{N} & 0.60 & 0.59 & 0.065 \\ & D_{O} & 0.35 & 0.32 & 0.070 \\ \mu_{-}^{\beta} & g & 0.40 & 0.41 & 0.057 \\ & D_{N} & 0.24 & 0.23 & 0.023 \\ \mu_{+}^{\beta} & D_{O} & 0.16 & 0.16 & 0.012 \\ & D_{N} & 0.07 & 0.07 & 0.010 \\ & D_{O} & 0.08 & 0.08 & 0.015\end{array}$

-0.0090
-0.0317
0.0089
-0.0088
-0.0026
-0.0008
-0.0097

0.0065

0.07

0.0065

0.08

0.0058

0.06

0.0021

0.02

0.0012

0.01

0.0010

0.01

0.015

0.0012

0.02 
Table 5 (continued)

\begin{tabular}{|c|c|c|c|c|c|c|c|}
\hline Par. & Proc. & True & Est. & RMSE & bias & SE & $\mathrm{SD}$ \\
\hline \multicolumn{8}{|c|}{ Model 6} \\
\hline \multirow[t]{3}{*}{$\mu^{\alpha}$} & $D_{N}$ & 0.60 & 0.56 & 0.095 & -0.0393 & 0.0092 & 0.12 \\
\hline & $D_{O}$ & 0.35 & 0.30 & 0.100 & -0.0478 & 0.0099 & 0.11 \\
\hline & $g$ & 0.40 & 0.40 & 0.075 & 0.0044 & 0.0083 & 0.09 \\
\hline \multirow[t]{3}{*}{$\mu_{-}^{\beta}$} & $D_{N}$ & 0.24 & 0.19 & 0.054 & -0.0414 & 0.0037 & 0.04 \\
\hline & $D_{O}$ & 0.16 & 0.14 & 0.034 & -0.0275 & 0.0022 & 0.03 \\
\hline & $g$ & 0.14 & 0.15 & 0.021 & 0.0104 & 0.0019 & 0.03 \\
\hline \multirow[t]{3}{*}{$\mu_{+}^{\beta}$} & $D_{N}$ & 0.07 & 0.06 & 0.018 & -0.0090 & 0.0015 & 0.02 \\
\hline & $D_{O}$ & 0.08 & 0.08 & 0.020 & -0.0080 & 0.0018 & 0.04 \\
\hline & $g$ & 0.17 & 0.17 & 0.024 & 0.0017 & 0.0025 & 0.03 \\
\hline
\end{tabular}

Note: Par. and Proc.: parameter type and process; True and Est.: true and estimated value; RMSE and BIAS: root mean squared error and bias of the estimates; SE: standard error of posterior medians across simulated datasets. SD: posterior standard deviation (mean across simulated datasets). Model 1 to 3 have constant guessing probabilities. Model 2 to 5 have suppressed process times for guessing "new" and model 3 and 5 also for guessing "old". $\mu^{\alpha}$ are transformed to process probabilities and $\mu_{-}^{\beta}$ and $\mu_{+}^{\beta}$ to process times in seconds

The last three models are not identified in the MPT setting without response times, and the process probability estimates are not as accurate as in the previous three. The largest difference between true and mean estimate is about 6\%. The corresponding RMSE value is 0.098 . Note, however, that goodness of recovery is comparable for the unconstrained Model 6 and the constrained Models 4 and 5. The process times are estimated as well or better for the constrained models than for the unconstrained model. The largest RMSE occurs for the unconstrained model with a value of about 0.055 . The largest RMSE for the constrained models is 0.031. Again, most of the biases (18 of 24) are slightly lower than zero, indicating that the true values are slightly underestimated (see Footnote 5).

For the restricted models, the mean of the estimated motor times differ from about $.7 \mathrm{~ms}$ to about $9.5 \mathrm{~ms}$ from the true values and have RMSE values ranging from about .009 to .015 . Their biases are slightly larger than zero (ranging from .0007 to .009), meaning they are slightly overestimated. In contrast, for the unrestricted model the mean of the two estimated motor times differ about 12 $\mathrm{ms}$ and $9 \mathrm{~ms}$ from the true values and have RMSE values of about .018 and .015 , respectively. The biases are also slightly larger than zero with values .009 and .012 , respectively.

The results of the recovery study suggest that the two features, holding process probabilities constant and suppressing process times, work quite well in terms of our evaluation criterion: Recovery was about as good or better for the models implementing the features as for the unconstrained model.

\section{General discussion}

Experience shows that the use of a new model such as the RT-MPT model class proposed by Klauer and Kellen (2018) hinges critically on the availability of user-friendly software. The purpose of this manuscript is to introduce and evaluate the $R$ package rtmpt that aims to provide such software. The only previous implementation of the RT-MPT model class relied on a proprietary software library and was available only in the form of $\mathrm{C}++$ code, severely limiting its accessibility. These shortcomings are overcome by $r t m p t$ that completely uses open source and free software, as well as the $R$ environment. Many changes in the underlying $\mathrm{C}++$ code were however necessary to accomplish these goals, calling for an attempt to demonstrate that the Bayesian algorithm has been implemented correctly.

The results of the validation studies raise our confidence that $r$ tmpt implements the Bayesian algorithm as intended. First and foremost, the rtmpt passed a strong and sensitive validity test in the form of simulation-based calibration. Moreover, rtmpt was able to recover the major results of analyses with the previous implementation by Klauer and Kellen (2018). Finally, a small recovery study suggested that two partially new features have been implemented correctly. 
These results and our thorough and repeated scrutiny of the program code sufficiently raise our confidence in the correctness of the implementation of the RT-MPT model class via rtmpt. We feel secure to offer the R package for public usage. Nevertheless, additional validation studies are desirable based on additional and more complex models. In this vein, we intend to implement the simulation-based calibration procedure as a general feature of rtmpt.

One reviewer suggested to make the SBC procedure available in the $R$ package. Therefore, we added two additional functions in rtmpt to simulate data (sim_rtmpt_data ()) and to run one replication of the SBC procedure (fit_rtmpt_SBC ()). The latter function in particular calculates the rank statistics for all the model parameters. These procedures are currently restricted to models such as the 2HTM with no more than two response categories for each tree. For more details, see the respective help files by entering

$>$ ?sim_rtmpt_data()

$>$ ?fit_rtmpt_SBC()

Is it possible to implement the RT-MPT model class in general-purpose software for Bayesian analyses such as Stan (Gelman et al., 2015) or JAGS (Plummer, 2003)? Being able to do so would come with considerable advantages. For example, prior distributions could be specified with more flexibility and variety than via rtmpt. Furthermore, an independent implementation, not relying on the original $\mathrm{C}++$ code, however much modified, would further decrease the probability of undetected errors if it were shown to perform comparable to the implementation based on the $\mathrm{C}++$ code. Accordingly, we invested considerable efforts into developing an implementation within Stan or JAGS.

As detailed in Klauer and Kellen (2018), the likelihood function of an RT-MPT model can be stated algebraically. Unfortunately, the likelihood is not differentiable, which is however a requirement in Stan. Therefore, implementing RT-MPT models in Stan was not possible. Another attempt consisted of implementing the model class via JAGS (Plummer, 2003). This entailed the construction of a socalled JAGS module (Wabersich \& Vandekerckhove, 2014) for those factors of the likelihood not already implemented in JAGS. We successfully built such a module, but the resulting MCMC algorithm did not converge reliably for reasons that are not clear to us. It is possible that these problems can be overcome, for example, by clever use of data augmentation steps, and we would very much welcome such a contribution.

As it stands, the current implementation provides an accessible tool to make use of the RT-MPT model class that has passed a number of validity tests. The MPT model class is popular and widely used. Moreover, in many research projects using MPT models response times are available over and above the responses themselves. Considering the advantages coming from modeling responses and response times jointly as outlined in the introduction, we are hoping for many researchers to take up the opportunity of using rtmpt for their purpose.

Acknowledgements This research was funded by the Deutsche Forschungsgemeinschaft (DFG) grant 2277, Research Training Group "Statistical Modeling in Psychology" (SMiP) as well as by a Koselleck grant, DFG K1 614/39-1, to the third author.

Author Note All $R$ scripts for the simulations and the empirical analysis, as well as experimental datasets, are available at the Open Science Framework: https://osf.io/9a4jw/.

\section{Appendix A: rtmpt functions for model restrictions and specifications}

The function set_params () takes four arguments. The first one is model, which is of the class rtmpt_model and names the model we want to alter. The second argument is parameters. This stands for the parameter we want to change-either "probs" for holding the probabilities constant, "tau_minus" for suppressing the process time(s) with negative outcome, or "tau_plus" for suppressing the process time(s) with positive outcome. The third argument is names and should be a character (vector) with the process name(s) to be changed. The last argument is values, which should be of the same length as names and provide numerical values; NA is used for estimating the given process parameters, values larger than zero and lower than one for holding process probabilities constant, and zero for suppressing a process time. Here are some examples:

- $>$ r2HTM <- set_params (model=r2HTM, parameters $=$ "probs", names="g", values=NA) will take the model $r 2 \mathrm{HTM}$, change the state of the probability parameter of the process ' ' $g$ ' ' to "to be estimated", and save it again in r $2 \mathrm{HTM}$,

- $>$ r2HTM <- set params (model=r2HTM, parameters="probs", names="g", values $=0.5$ ) will take the model $r 2 \mathrm{HTM}$, change the probability parameter of the process ' ' $\mathrm{g}$ ' ' to the constant 0.5 , and save it again in $r 2 \mathrm{HTM}$,

- $>$ r2HTM <- set_params (model=r2HTM, parameters="tau_minus", names="g", values $=0$ ) will take the model $r 2 \mathrm{HTM}$, change the completion-time parameter $\tau_{g}^{-}$of the process ' ' $g$ ' ' to 0 (meaning it will be suppressed), and save it again in $r 2 \mathrm{HTM}$. 
The function set resps () takes four arguments. The first is again the model to be altered. The second is tree, which should be the label of the tree, for which we want to make a change, as a character (or number if numbers are used in the model). Third is categories which specifies the categories within the tree, for which we want to make changes. This should again be a vector of characters (or numbers if numbers are used in the model). The last argument values must be a vector of numbers corresponding to a certain response. For example,

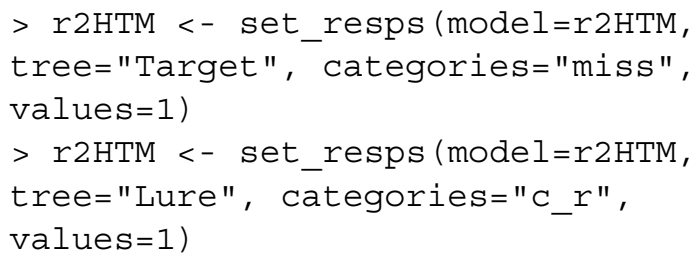

will define a separate motor time label for all "new" responses if it was not already taken care of in the $E Q N$ or $M D L$ file. This means, we would assume different motor time distributions for "old" and "new" responses.

\section{Appendix B: Prior specifications in rtmpt}

The settings concern the prior distributions of

- $\mu^{(\alpha)}$, the group-level means of the probit-transformed process probabilities (2), which are given a normal prior distribution with mean 0 ,

- $\mu^{(\beta)}$, the group-level means of the log-transformed process rates (2) with a gamma prior on the (original) exponential scale,

- $\Sigma$, the variance-covariance matrix estimated for the probit-transformed process probabilities $\boldsymbol{\alpha}_{s}$ and the logtransformed process rates $\boldsymbol{\beta}_{s}$ from Eq. 2 with a scaled inverse Wishart prior distribution,

- $\mu^{(\gamma)}$, the group-level means of the motor times (2) with a normal distribution as prior,

- $\Gamma$, the variance-covariance matrix estimated for the mean person-level motor times, $\boldsymbol{\gamma}_{s}$, from Eq. 2 with a scaled inverse Wishart prior distribution (Gelman et al., 2014),

- $\sigma_{s}^{2}$, the person-level variances of motor times (1), with a scaled inverse Chi-squared distribution with scale factor $\omega^{2}$, and

- $\omega^{2}$, which can be interpreted as an estimate of the overall residual variance and is given a gamma prior.
The prior distributions of $\Sigma$ and $\Gamma$ are scaled inverse Wishart distributions with $d f$ degrees of freedoms as specified in Klauer and Kellen (2018). A matrix follows this distribution if it is distributed as $\xi^{T} A \xi$, with scale factors $\xi$ distributed normally with mean 1 and prior variance $\epsilon^{-1}$, and $A$ distributed as an inverse Wishart distribution with scale matrix $E$. The scale factors $\xi$ permit the variances in $\Sigma$ and $\Gamma$ to be estimated with fewer constraints than imposed by the unscaled Wishart distribution (Gelman et al., 2014). The user can set their variance, or rather their precision $\epsilon$; the default value is 1.0. Choosing smaller precision values results in less informative priors for the variances, choosing larger values results in priors more in line with the unscaled inverse Wishart distribution. Precision parameter $\epsilon$, labelled prec_epsilon in the prior_params list argument of the function fit_rtmpt (), also applies to the variances of $\mu^{(\alpha)}$. The default value 1.0 implies a uniform prior distribution of these parameters on the original probability scale. Again, smaller precision values make their prior distributions even less informative, whereas larger values will make the prior distributions more focused around the mean value of 0.5 on the probability scale.

The just-mentioned inverse Wishart distributions also require a scale matrix $E$, which is the identity matrix by default. But via the list elements sf_of_scale_matrix_SIGMA and sf_of_scale_matrix_GAMMA of the prior_params list, the identity matrix can be scaled by a constant for $\Sigma$ and $\Gamma$, respectively. Values smaller than the default 1.0 imply smaller a priori variances and less variability in samples from the prior distribution for $\Sigma$ and $\Gamma$, values larger than 1.0 imply larger a priori variances and permit more variability in samples from the resulting prior distribution.

Additionally, the degrees of freedom $d f$ of the inverse Wishart distribution underlying the priors for $\Sigma$ and $\Gamma$ can be adjusted. $d f$ is by default the number of parameters plus $k$ with $k$ being 1 . This ensures a priori uniformly distributed correlations between -1 and 1 corresponding to an uninformative prior distribution. With the rtmpt parameter add df to invWish the user can change the value of $k$. With larger $k$, the expected values of the variances and covariances get closer to zero (Gelman et al., 2014).

The user can also specify mean and variance of $e^{\mu^{(\beta)}}$, the Gamma distributed group-level estimates of the process rates. For simplicity the user can set the mean and variance, instead of shape and rate, of the Gamma distribution via mean_of_exp_mu_beta and var_of_exp_mu_beta in the prior_params list. Note that shape and rate can be transformed unambiguously into mean and variance for 
Table 6 Overview of prior parameters to set in the prior_params argument of the fit_rtmpt () function

\begin{tabular}{|c|c|c|c|}
\hline Name & Description & Default & Restriction \\
\hline prec_epsilon & Precision of the normal distribution for $\mu^{(\alpha)}$ and $\xi$ & 10 & $>0$ \\
\hline mean_of_exp_mu_beta & Group-specific mean of the exponential rates & 10 & $>0$ \\
\hline var_of_exp_mu_beta & Group-specific variance of the exponential rates & 100 & $>0$ \\
\hline mean_of_mu_gamma & Group-specific mean of the motor times & 0 & \\
\hline var_of_mu_gamma & Group-specific variance of the motor times & 10 & $>0$ \\
\hline mean_of_omega_sqr & Mean of the residual variance & 0.005 & $>0$ \\
\hline var_of_omega_sqr & Variance of the residual variance & 0.01 & $>0$ \\
\hline df_of_sigma_sqr & $\begin{array}{l}\text { Degrees of freedom for the individual variance of the response } \\
\text { executions }\end{array}$ & 2 & $\geqslant 2$ \\
\hline sf_of_scale_matrix_SIGMA & Scaling factor of $\Sigma$ & 1 & $>0$ \\
\hline sf_of_scale_matrix_GAMMA & Scaling factor of $\Gamma$ & 1 & $>0$ \\
\hline add_df_to_invWish & $\begin{array}{l}\text { Additional degrees of freedom (integer) in the scaled inverse } \\
\text { Wishart distribution of } \Sigma \text { and } \Gamma\end{array}$ & 1 & $>0$ \\
\hline
\end{tabular}

the Gamma distribution. By default, these are given values of 10 and 100, implying a mean process time in the order of $(1 / 10) \mathrm{s}=.1 \mathrm{~s}$ with a large prior variance. ${ }^{6}$

Similarly, the prior mean and variance of $\mu^{(\gamma)}$ (normally distributed group-specific mean of the person-level motor-times $\boldsymbol{\gamma}_{s}$ ) can be set via mean_of_mu_gamma and var_of_mu_gamma of the prior_params list. Their default setting is $0 \mathrm{~s}$ and 10 , respectively, which are reasonable values for the applications we studied so far, but can be adjusted according to the motor times expected in a given application and the amount of uncertainty that exists about them a priori.

Furthermore, the degrees of freedom for the prior distribution of $\sigma_{s}^{2}$, the person-level variances of motor times, can be set. The default setting is 2, resulting in a relatively uninformative prior distribution. If little

\footnotetext{
${ }^{6}$ Remember that process times are exponentially distributed with rate parameter $e^{\mu^{(\beta)}}$ and the inverse of the rate parameter is the expected value of the process times. These settings seem reasonable for many applications, but when processes are expected to take much longer or much shorter to complete, the user is free to adjust the mean values and via the variance the prior uncertainty in the mean settings.
}

heterogeneity between participants in the variability of motor times is expected, degrees of freedom can be set to higher values to reflect this, using df_of_sigma_sqr in prior_params list.

The scale factor of the scaled inverse $\chi^{2}$ distribution of $\sigma_{s}^{2}$ is $\omega^{2}$, the residual variance in response times, not accounted for by the RT-MPT model. The residual variance is Gamma distributed and its prior mean and variance, instead of shape and rate, can be specified via mean_of_omega_sqr and var_of_omega_sqr, respectively of the prior_params list argument. By default, values of 0.005 and 0.01 are set on the basis of our experience using the model, implying a prior expectation of the mean residual variance in the order of 0.005 for response times specified in seconds with a reasonably large amount of a priori variability or uncertainty.

Table 6 lists all parameters of the prior_params argument list of the function fit rtmpt (). The table shows the name of the usable list elements, a short description, default values, and the restrictions for setting their values. The $R$ help file for the fit_rtmpt () function provides additional information on this named list argument prior_params. 


\section{Appendix C: Outputs for model and data transformation}

Listing 1 and 2 show the respective outputs of the model and the data transformation.

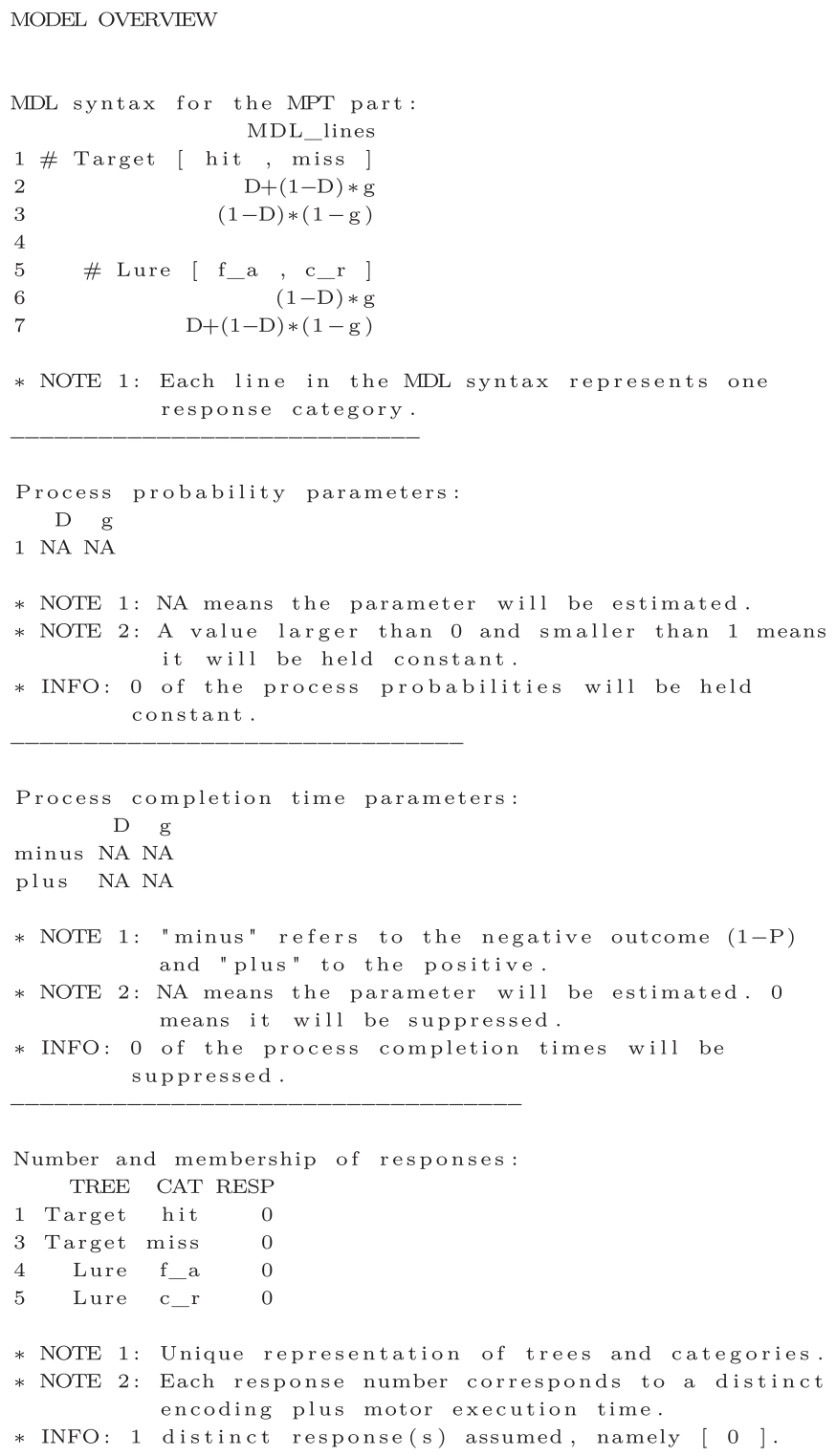

Listing 1 Example model output via the $R$ function to_rtmpt_model () 
DATA TRANSFORMATION OVERVIEW

Reordered variables:

subj, group, tree, cat, rt

* NOTE1: Additional variables are attached behind these five.

* NOTE2: To see your data frame use <object name>\$data.

Transformed variable (s):

"subj"

$\begin{array}{rrr} & \text { old } & \text { new } \\ & 1 & 0 \\ & 2 & 1 \\ & 3 & 2 \\ & 4 & 3 \\ & 5 & 4 \\ & 6 & 5 \\ & 7 & 6 \\ & 8 & 7 \\ & 9 & 8 \\ 0 & 10 & 9 \\ 1 & 11 & 10 \\ 2 & 12 & 11 \\ 3 & 13 & 12 \\ 4 & 14 & 13 \\ 5 & 15 & 14 \\ 6 & 16 & 15 \\ 7 & 17 & 16 \\ 8 & 18 & 17 \\ 9 & 19 & 18 \\ 0 & 20 & 19 \\ 1 & 21 & 20 \\ 2 & 22 & 21 \\ 3 & 23 & 22 \\ 4 & 24 & 23 \\ 5 & 25 & 24 \\ 6 & 26 & 25 \\ 7 & 27 & 26 \\ 8 & 28 & 27 \\ 9 & 29 & 28 \\ 0 & 30 & 29 \\ 1 & 31 & 30 \\ 2 & 32 & 31 \\ 3 & 33 & 32 \\ 4 & 34 & 33 \\ 5 & 35 & 34 \\ 6 & 36 & 35 \\ 7 & 37 & 36 \\ 8 & 38 & 37 \\ 9 & 39 & 38 \\ 0 & 40 & 39 \\ & & \end{array}$

" group "

old new

$1 \quad 1 \quad 0$

"tree"

old new

1 Target 0

2 Lure

" cat "

old new

1 hit 0

2 miss 1

3 f a 2

4 c_r 3

* NOTE: "old" refers to the used labels and "new" to the ones that will be used.

Listing 2 Example output of the data transformation via the $R$ function to_rtmpt_data () 


\section{Appendix D: Prior specification for the simulation-based calibration study}

Since the original priors used in the program are too broad and lead to too many extreme RTs (RTs from zero to over $10,000 \mathrm{~ms}$ ) and categories with no observations, some adjustments were required. First, the precision parameter $\epsilon$ for $\mu^{(\alpha)}$ (probit-transformed group-level process probabilities) and $\xi$ (scale parameter for the scaled inverse Wishart distribution of the variance-covariance matrices) is increased from 1 to 10 to ensure that probability parameters are almost never zero or one and therefore the number of categories with zero observations is small. This also ensures that $\xi$ is not too large, which is important because it can cause too large values in $\Sigma$ and $\Gamma$, the variances-covariance matrices. Second, mean and variance of the Gamma distribution for $e^{\mu^{(\beta)}}$ (group-mean process rate) are changed from 10 and 100 to 10 and 10 , respectively. Since the process times are exponentially distributed a group-mean rate of 10 corresponds to a mean process time of 100 milliseconds. The choice of the variance ensures that most of the group-mean process times are between 50 and 250 milliseconds. Third, the mean and variance parameter of $\mu^{(\gamma)}$ are changed from 0 and 10 to .5 and .0025 , respectively, which ensures that most values of $\mu^{(\gamma)}$ fall roughly between 350 and 650 milliseconds. As a reminder, this is not the mean of the encoding and motor execution time $\delta$, since $\delta \sim \mathcal{T N}(a=0, b=$ $\infty, \mu=\mathbb{E}[\gamma], \sigma^{2}=\sigma^{2}$ ), where $\mathbb{E}[\gamma]=\mathbb{E}\left[\mu^{(\gamma)}\right]=.5$ seconds, which means the actual expected encoding and motor execution time is higher. Fourth, we changed the prior distributions of two related parameters, $\omega^{2}$ (residual variance) and $\sigma^{2}$ (variance of the motor times). Parameter $\omega^{2}$ follows a gamma distribution with shape and rate set to values, such that the mean is .005 and the variance $4.0 e-6$. Parameter $\sigma^{2}$ follows a scaled inverse $\chi^{2}$ distribution with 10 degrees of freedom (instead of two) and $\omega^{2}$ as scale parameter. Since the mean of the overall residual parameter $\omega^{2}$ is .005 the expected value of the $\sigma^{2}$ is .00625 . Finally, the variance-covariance matrices, $\Sigma$ and $\Gamma$, have scaled inverse Wishart priors. The first with $p+5$ degrees of freedom, scale matrix $\left(.1 \times \boldsymbol{I}_{p}\right)$, and scale parameter $\xi$, where $p$ is the number of process parameters (in our case six: two probability and four process rate/time parameters). The second has $r+5$ degrees of freedom, scale matrix $\left(.01 \times \boldsymbol{I}_{r}\right)$, and also scale parameter $\xi$, where $r$ is the number of responses (in our case two). This leads to less extreme correlations and variances. The reason for the difference in the scale matrices is that the process-related parameters are on a different scale than the encoding and motor execution times.

\section{References}

Arnold, N. R., Bröder, A., \& Bayen, U. J. (2015). Empirical validation of the diffusion model for recognition memory and a comparison of parameter-estimation methods. Psychological Research Psychologische Forschung, 79, 882-898. https://doi.org/10.1007/s00426-014-0608-y

Asendorpf, J. B., Conner, M., De Fruyt, F., De Houwer, J., Denissen, J. J. A., Fiedler, K., \& Wicherts, J. M. (2013). Recommendations for increasing replicability in psychology. European Journal of Personality, 27, 108-119. https://doi.org/10.1002/per.1919

Batchelder, W. H., \& Riefer, D. M. (1999). Theoretical and empirical review of multinomial process tree modeling. Psychonomic Bulletin \& Review, 6, 57-86. https://doi.org/10.3758/bf03210812

Donders, F. C. (1868). Over de snelheid van psychische processen. Onderzoekingen gedaan in het Physiologisch Laboratorium der Utrechtsche Hoogeschool (1968-1869), 2, 92-120.

Dube, C., Starns, J. J., Rotello, C. M., \& Ratcliff, R. (2012). Beyond ROC curvature: Strength effects and response time data support continuous-evidence models of recognition memory. Journal of Memory and Language, 67, 389-406. https://doi.org/10.1016/j. jml.2012.06.002.

Erdfelder, E., Auer, T. S., Hilbig, B. E., Aßfalg, A., Moshagen, M., \& Nadarevic, L. (2009). Multinomial processing tree models: A review of the literature. Zeitschrift für Psychologie/Journal of Psychology, 217, 108-124. https://doi.org/10.1027/0044-3409.217. 3.108 .

Galassi, M., Davies, J., Theiler, J., Gough, B., Jungman, G., Alken, P., \& Ulerich, R. (2018). GNU scientific library [Computer software]. http://www.gnu.org/software/gsl/.

Gelman, A., Carlin, J., Stern, H., Dunson, D., Vehtari, A., \& Rubin, D. (2014). Bayesian data analysis, (3rd ed.). Boca Raton: Taylor \& Francis Group.

Gelman, A., Lee, D., \& Guo, J. (2015). Stan: A probabilistic programming language for Bayesian inference and optimization. Journal of Educational and Behavioral Statistics, 40, 530-543. https://doi.org/10.3102/1076998615606113

Gelman, A., \& Rubin, D. B. (1992). Inference from iterative simulation using multiple sequences. Statistical Science, 7, 457472. https://doi.org/10.1214/ss/1177011136

Heck, D. W., \& Erdfelder, E. (2016). Extending multinomial processing tree models to measure the relative speed of cognitive processes. Psychonomic Bulletin \& Review, 23, 1440-1465. https://doi.org/10.3758/s13423-016-1025-6

Heck, D. W., \& Erdfelder, E. (in press). Benefits of response timeextended multinomial processing tree models: A reply to Starns (2018). Psychonomic Bulletin \& Review.

Heck, D. W., Arnold, N. R., \& Arnold, D. (2018a). TreeBUGS: An $\mathrm{R}$ package for hierarchical multinomial-processing-tree modeling. Behavior Research Methods, 50, 264-284. https://doi.org/10. 3758/s13428-017-0869-7.

Heck, D. W., Erdfelder, E., \& Kieslich, P. J. (2018b). Generalized processing tree models: Jointly modeling discrete and continuous variables. Psychometrika, 1-26. https://doi.org/10.1007/s11336018-9622-0.

Hu, X. (1999). Multinomial processing tree models: An implementation. Behavior Research Methods, Instruments \& Computers, 31, 689-695. https://doi.org/10.3758/bf03200747

$\mathrm{Hu}, \mathrm{X}$. (2001). Extending general processing tree models to analyze reaction time experiments. Journal of Mathematical Psychology, 45, 603-634. https://doi.org/10.1006/jmps.2000.1340

Hütter, M., \& Klauer, K. C. (2016). Applying processing trees in social psychology. European Review of Social Psychology, 27, 116-159. https://doi.org/10.1080/10463283.2016.1212966 
Jacoby, L. L. (1991). A process dissociation framework: Separating automatic from intentional uses of memory. Journal of Memory and Language, 30, 513-541. https://doi.org/10.1016/0749-596x (91)90025-f.

Klauer, K. C. (2010). Hierarchical multinomial processing tree models: A latent-trait approach. Psychometrika, 75, 70-98. https://doi.org/10.1007/s11336-009-9141-0

Klauer, K. C., \& Kellen, D. (2018). RT-MPTs: Process models for response-time distributions based on multinomial processing trees with applications to recognition memory. Journal of Mathematical Psychology, 82, 111-130. https://doi.org/10.1016/j.jmp.2017.12. 003.

Plummer, M. (2003). JAGS: A program for analysis of Bayesian graphical models using Gibbs sampling [Computer software]. http://mcmc-jags.sourceforge.net/.

Plummer, M., Best, N., Cowles, K., \& Vines, K. (2006). Coda: Convergence diagnosis and output analysis for MCMC. R News, 6, 7-11. https://journal.r-project.org/archive/.

R Core Team (2019). R: A language and environment for statistical computing [Computer software manual]. https://www.R-project. org/.

Ratcliff, R. (1978). A theory of memory retrieval. Psychological Review, 85, 59. https://doi.org/10.1037/0033-295x.85.2.59

Riefer, D. M., \& Batchelder, W. H. (1988). Multinomial modeling and the measurement of cognitive processes. Psychological Review, 95, 318. https://doi.org/10.1037/0033-295x.95.3.318

Singmann, H., \& Kellen, D. (2013). MPTinR: Analysis of multinomial processing tree models in R. Behavior Research Methods, 45, 560-575. https://doi.org/10.3758/s13428-012-0259-0

Snodgrass, J. G., \& Corwin, J. (1988). Pragmatics of measuring recognition memory: Applications to dementia and amne- sia. Journal of Experimental Psychology: General, 117, 34. https://doi.org/10.1037/0096-3445.117.1.34

Spiegelhalter, D. J., Best, N. G., Carlin, B. P., \& Van Der Linde, A. (2002). Bayesian measures of model complexity and fit. Journal of the Royal Statistical Society: Series B (Statistical Methodology), 64, 583-639. https://doi.org/10.1111/1467-9868.00353

Starns, J. J. (2018). Adding a speed-accuracy trade-off to discrete-state models: A comment on Heck and Erdfelder (2016). Psychonomic Bulletin \& Review, 25, 2406-2416. https://doi.org/10.3758/s13423-018-1456-3

Talts, S., Betancourt, M., Simpson, D., Vehtari, A., \& Gelman, A. (2018). Validating Bayesian inference algorithms with simulationbased calibration. arXiv:1804.06788.

The Numerical Algorithms Group (NAG) (2017). The NAG C library [Computer software]. http://www.nag.com.

Vehtari, A., Gelman, A., \& Gabry, J. (2017). Practical Bayesian model evaluation using leave-one-out cross-validation and WAIC. Statistics and Computing, 27, 1413-1432. https://doi.org/10.1007/ s11222-016-9696-4.

Wabersich, D., \& Vandekerckhove, J. (2014). Extending jags: A tutorial on adding custom distributions to JAGS (with a diffusion model example). Behavior research methods, 46, 15-28. https://doi.org/10.3758/s13428-013-0369-3

Watanabe, S. (2010). Asymptotic equivalence of Bayes cross validation and widely applicable information criterion in singular learning theory. Journal of Machine Learning Research, 11, 35713594.

Publisher's note Springer Nature remains neutral with regard to jurisdictional claims in published maps and institutional affiliations. 